\title{
ON THE SYMMETRIC CUBE OF A SPHERE
}

\author{
BY \\ JACK UCCI
}

1. Introduction. Let $X^{m}$ denote the cartesian product $X \times \cdots \times X$ ( $m$ factors) of the based space $X$. The full symmetric group $S(m)$ acts on $X^{m}$ by permutation homeomorphisms and the quotient space $X^{m} / S(m)$ is defined to be the $m$-fold symmetric product $S P^{m} X$ of $X$.

Now let $X=S^{n}$, the $n$-sphere. A map $f: S P^{m} S^{n} \rightarrow S^{n}$ is of type $r$ if the composite

$$
S^{n} \stackrel{i}{\longrightarrow} S P^{m} S^{n} \stackrel{f}{\longrightarrow} S^{n}
$$

has degree $r$. Here $i(x)=[x, e, \ldots, e]$ where $e$ is the basepoint of $S^{n}$. For given $n$ and $m$ an elementary result of James asserts that the set of all "realizable types" is an ideal $\left(k^{m, n}\right) \subset Z$ in the ring of integers-whence the problem of determining the generator $k^{m, n}$. The main results of [1] and [6] determine $k^{2, n}$ :

THEOREM 1.1. (i) $k^{2,2 t}=0$;

(ii) $k^{2,2 t+1}=2^{\varphi(2 t)}$ where $\varphi(b)$ is the number of integers $0<a \leqq b$ with $a \equiv 0,1,2$ or $4 \bmod 8$.

In [7] we obtained a lower bound for $k^{m, n}$ for all $m$. Our main result in this paper asserts that this lower bound is best possible when $m=3$, i.e. $k^{3, n}$ is determined as follows:

THEOREM 1.2. (i) $k^{3,2 t}=0$; (ii) $k^{3,2 t+1}=2^{\varphi(2 t)} \cdot 3^{t}$.

1.2(i) is of course a simple consequence of 1.1(i). Moreover the main result of [7] implies that $2^{\varphi(2 t)} \cdot 3^{t} \mid k^{3,2 t+1}$. Thus $1.2($ ii) will be proved by constructing a map $f: S P^{3} S^{n} \rightarrow S^{n}, n=2 t+1$, of type $r=2^{\varphi(2 t)} \cdot 3^{t}$. For this Toda's notion of the suspension-order of a space $X$-the least positive integer $s$ such that $s \iota_{E X}=0$ in $[E X, E X]$-is fundamental (see $\S 4)$.

Recall then Toda's result [6] which is an important step in the proof of 1.1(ii). Let $X_{2,1}^{n}$ be the quotient space $X / S(2)$ where $X$ is the $S(2)$-invariant subspace $D^{n} \times S^{n-1} \cup S^{n-1} \times D^{n}$ of $\left(D^{n}\right)^{2}$. Then $X_{2,1}^{n}$ has the homotopy type of a suspension space (see Corollary 2.7 or [1, Lemma 2.1]) so that we may consider the "desuspension" $E^{-1} X_{2,1}^{n}$ of $X_{2,1}^{n}$. Toda's result asserts

THEOREM 1.3. (i) The suspension-order of $E^{-1} X_{2,1}^{2 t}$ is infinite.

(ii) The suspension-order of $E^{-1} X_{2,1}^{2 t-1}$ is $2^{\varphi(2 t)}$.

Received by the editors November 26, 1968. 
In a similar way 1.2(ii) depends on a suspension-order calculation. Let $X_{3,2}^{n}$ be the quotient space $Y / S(3)$ where $Y$ is the $S(3)$-invariant subspace $D^{n} \times S^{n-1}$ $\times S^{n-1} \cup S^{n-1} \times D^{n} \times S^{n-1} \cup S^{n-1} \times S^{n-1} \times D^{n}$ of $\left(D^{n}\right)^{3}$. The standard embedding $\left(D^{n}\right)^{2} \rightarrow\left(D^{n}\right)^{3},\left(x_{1}, x_{2}\right) \rightarrow\left(x_{1}, x_{2}, e\right), e$ the basepoint of $D^{n}$, induces an embedding $X_{2,1}^{n} \rightarrow X_{3,2}^{n}$. The quotient space $X_{3,2}^{n} / X_{2,1}^{n}$ has the homotopy type of a suspension space (see Proposition 2.6). Then Toda's methods can be applied to prove

THEOREM 1.4. (i) The suspension-order of $E^{-1}\left(X_{3,2}^{2 t} / X_{2,1}^{2 t}\right)$ is infinite.

(ii) The suspension-order of $E^{-1}\left(X_{3,2}^{2 t+1} / X_{2,1}^{2 t+1}\right)$ is $3^{t}$.

Apart from some cohomological calculations our procedure is that of $\S 4$ of [1]namely killing a certain attaching map by composition with a suitable map. In $\S 1$ we develop some geometry of $S P^{m} S^{n}$. In particular we study certain desuspension properties of related subcomplexes. $\$ 2$ contains a lemma sharpening our focus on exactly which map should be killed. 1.4 and 1.3 are then proved in $\S 3$ and $\S 4$, respectively. The latter makes use of the mod $3 \mathrm{Hopf}$ invariant.

Given a space $Y$ many properties of its suspension $E Y$, e.g. the group structure on $[E Y, Z]$, the suspension-order of $Y$ (which is a property defined in terms of $E Y$ ), etc. admit definitions for any space $X$ of the same homotopy type as $E Y$. Thus we often speak of these properties for $E^{-1} X$ when we really mean the corresponding properties for $Y$ (or $E Y$ ), even though we don't know if $X$ is actually desuspendable.

Most of this paper was written at the Forschungsinstitut für Mathematik, Eidgenössosche Technische Hochschule in Zürich, during the summer of 1968. It is a pleasure to thank Professor Beno Eckmann for making my visit possible.

2. Geometry of $S P^{m} S^{n}$. Let $D^{n}$ be the unit $n$-ball in $R^{n}$ and $S^{n-1}=\partial D^{n}$ its boundary $(n-1)$-sphere. Let $h_{\tau}:\left(D^{n}\right)^{m} \rightarrow\left(D^{n}\right)^{m}$ be the permutation homeomorphism corresponding to a given element $\tau \in S(m)$. Setting $A_{m, l}^{n}=\left(D^{n}\right)^{m-l}$ $\times\left(S^{n-1}\right)^{l}, 0 \leqq l \leqq m$, we obtain an $S(m)$-invariant subspace of $A_{m, 0}^{n}=\left(D^{n}\right)^{m}$ by

$$
\tilde{X}_{m, l}^{n}=\bigcup_{\tau \in S(m)} h_{\tau}\left(A_{m, l}^{n}\right)
$$

Hence we may take its orbit space under the $S(m)$-action and obtain, $X_{m, l}^{n}=$ $\tilde{X}_{m, l}^{n} / S(m)$.

We give $X_{m, l}^{n}$ the quotient topology. The embedding $A_{m^{\prime}, l^{\prime}}^{n} \rightarrow A_{m, l}^{n}, m^{\prime} \leqq m$ and $m^{\prime}-l^{\prime} \leqq m-l$, given by $\left(x_{1}, \ldots, x_{m^{\prime}}\right) \rightarrow\left(x_{1}, \ldots, x_{m^{\prime}}, e, \ldots, e\right)$ induces an embedding $X_{m^{\prime}, l^{\prime}}^{n} \rightarrow X_{m, l}^{n} . X_{m, 0}^{n}$ and $X_{m, m}^{n}$ are the familiar symmetric products $S P^{m} D^{n}$ and $S P^{m} S^{n-1}$, respectively. And $X_{2,1}^{n}$ is the "symmetric join" of $S^{n-1}$ with itself defined in [1].

LEMMA 2.1. $X_{m, 0}^{n}$ is homeomorphic to the cone

$$
C X_{m, 1}^{n}=X_{m, 1}^{n} \times I / X_{m, 1}^{n} \times\{0\} .
$$


Proof. The map $X_{m, 1}^{n} \times I \rightarrow X_{m, 0}^{n}$ given by $([x], t)=\left(\left[x_{1}, \ldots, x_{m}\right], t\right) \rightarrow[t x]$ $=\left[t x_{1}, \ldots, t x_{n}\right]$ induces a topological map

$$
C X_{m, 1}^{n} \stackrel{\alpha}{\longrightarrow} X_{m, 0}^{n}
$$

which sends $X_{m, 1}^{n} \times\{1\}$ onto the subspace $X_{m, 1}^{n} \subset X_{m, 0}^{n}$.

RemarK. All cones $C X$ in this paper will be "inverted", i.e. $C X=X \times I / X \times\{0\}$. As we define the suspension $E X$ to to be the quotient $C X / X=C X / X \times\{1\}$, our suspensions will also be inverted.

Choose a relative homeomorphism $h:\left(D^{n}, S^{n-1}\right) \rightarrow\left(S^{n}, e\right)$. Then the induced map

$$
h_{S(m)}:\left(X_{m, 0}^{n}, X_{m, 1}^{n}\right) \rightarrow\left(X_{m, m}^{n+1}, X_{m-1, m-1}^{n+1}\right)
$$

also is a relative homeomorphism and so from Lemma 2.1 we have

Lemma 2.2. $X_{m, m}^{n+1}$ is homeomorphic to the adjunction space $X_{m-1, m-1}^{n+1} \cup C X_{m, 1}^{n}$.

Here the attaching map is given by the restriction of $h_{S(m)}$ to $X_{m, 1}^{n}$.

We define the join $X \circ Y$ of $X$ and $Y$ as the space $X \times C Y \cup C X \times Y$. The homeomorphism $c: C(X \circ Y) \rightarrow C X \times C Y$ given by

$$
\begin{array}{ll}
c[(x,[y, t]), u]=[[x, u],[y, t u]] & \text { if }(x,[y, t]) \in X \times C Y, \\
c[([x, t], y), u]=[[x, t u],[y, u]] & \text { if }([x, t], y) \in C X \times Y
\end{array}
$$

induces a homeomorphism

$$
E(X \circ Y)=\frac{C(X \circ Y)}{X \circ Y} \stackrel{\hat{c}}{\longrightarrow} \frac{C X \times C Y}{X \circ Y} .
$$

Consider the shuffle map $\beta: X_{k, l}^{n} \times X_{k^{\prime}, l^{\prime}}^{n} \rightarrow X_{k+k^{\prime}, l+l^{\prime}}^{n}$ defined by

$$
\beta\left(\left[x_{1}, \ldots, x_{k}\right],\left[y_{1}, \ldots, y_{k^{\prime}}\right]\right)=\left[x_{1}, \ldots, x_{k}, y_{1}, \ldots, y_{k^{\prime}}\right] \text {. }
$$

Using the homeomorphism (1) we obtain a map

$$
\psi: X_{k, 1}^{n} \circ X_{k^{\prime}, 1}^{n} \rightarrow X_{k+k^{\prime}, 1}^{n}
$$

by setting $\psi \mid X_{k, 1}^{n} \times C X_{k^{\prime}, 1}^{n}=\beta \circ(\mathrm{id} \times \alpha)$ and $\psi \mid C X_{k, 1}^{n} \times X_{k^{\prime}, 1}^{n}=\beta \circ(\alpha \times \mathrm{id})$. The maps $\alpha, \beta, \psi$ and $c$ satisfy the commutative diagram

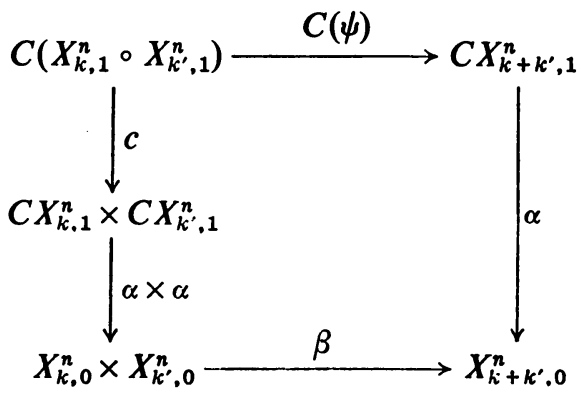


Here $C(\psi)$ denotes the conal extension of the map $\psi$. The verification of the commutativity is straightforward: for $(x,[y, t]) \in X_{k, 1}^{n} \times C X_{k^{\prime}, 1}^{n}, x=\left[x_{1}, \ldots, x_{k}\right]$, $y=\left[y, \ldots, y_{k^{\prime}}\right]$ we have

$$
\begin{gathered}
\alpha \circ C(\psi)[(x,[y, t]), u]=\alpha[\psi(x,[y, t]), u]=\alpha[\beta(x, t y), u]=u \beta(x, t y)=[u x, t u y], \\
\beta \circ(\alpha \times \alpha) \circ c[(x,[y, t]), u]=\beta \circ(\alpha \times \alpha)[[x, u],[y, t u]]=\beta(u x, t u y)=[u x, t u y]
\end{gathered}
$$

Similarly for $([x, t], y) \in C X_{k, 1}^{n} \times X_{k^{\prime}, 1}^{n}$. As an immediate consequence we have

COROLlary 2.3. The shuffle map $\beta: X_{m, 0}^{n} \times X_{m^{\prime}, 0}^{n} \rightarrow X_{m+m^{\prime}, 0}^{n}$ induces the suspension map

$$
E\left(X_{m, 1}^{n} \circ X_{m^{\prime}, 1}^{n}\right) \stackrel{E \psi}{\longrightarrow} X_{m+m^{\prime}, 0}^{n} / X_{m+m^{\prime}, 1}^{n} \cong E X_{m+m^{\prime}, 1}^{n}
$$

Now consider the surjective map $X_{m-l, 0}^{n} \times X_{l, 0}^{n-1} \stackrel{\alpha}{\rightarrow} X_{m, l}^{n}, 1 \leqq l<m$, defined by the composite

$$
X_{m-l, 0}^{n} \times X_{l, 0}^{n-1} \stackrel{\mathrm{id} \times h_{S(l)}}{\longrightarrow} X_{m-l, 0}^{n} \times X_{l, l}^{n} \stackrel{\beta}{\longrightarrow} X_{m, l}^{n}
$$

where $h_{S(l)}$ is given in (2) and $\beta$ is the shuffle map. It is easy to check that the boundary of $X_{m-l, 0}^{n} \times X_{l, 0}^{n-1}$

$$
\partial\left(X_{m-l, 0}^{n} \times X_{l, 0}^{n-1}\right) \cong \partial\left(C X_{m-l, 1}^{n} \times C X_{l, 1}^{n-1}\right)=X_{m-l, 1}^{n} \circ X_{l, 1}^{n-1}
$$

is mapped by $d$ onto the subspace $X_{m, l+1}^{n} \cup X_{m-1, l-1}^{n}$ and that $d$ defines a relative homeomorphism of the pairs

$$
\left(X_{m-l, 0}^{n} \times X_{l, 0}^{n-1}, X_{m-l, 1}^{n} \circ X_{l, 1}^{n-1}\right) \text { and }\left(X_{m, l}^{n}, X_{m, l+1}^{n} \cup X_{m-1, l-1}^{n}\right) \text {. }
$$

And so $d c$ defines a relative homeomorphism of the pairs

$$
\left(C\left(X_{m-l, 1}^{n} \circ X_{l, 1}^{n-1}\right), X_{m-l, 1}^{n} \circ X_{l, 1}^{n-1}\right) \text { and }\left(X_{m, l}^{n}, X_{m, l+1}^{n} \cup X_{m-1, l-1}^{n}\right) .
$$

Note $c \mid\left(X_{m-l, 1}^{n} \circ X_{l, 1}^{n-1}\right)$ is the identity map.

Similarly $\beta: X_{m-l, 0}^{n} \times X_{l, l}^{n} \rightarrow X_{m, l}^{n}$ itself is a surjective map which defines a relative homeomorphism of the pairs

$$
\left(X_{m-l, 0}^{n} \times X_{l, l}^{n}, X_{m-l, 1}^{n} \times X_{l, l}^{n}\right) \text { and }\left(X_{m, l}^{n}, X_{m, l+1}^{n}\right) .
$$

Summarizing these observations we have

LEMMA 2.4. Let $1 \leqq l<m$. Then

(i) $X_{m, l}^{n}$ is homeomorphic to the adjunction space $\left(X_{m, l+1}^{n} \cup X_{m-1, l-1}^{n}\right) \cup$ $C\left(X_{m-l, 1}^{n} \circ X_{l, 1}^{n-1}\right)$ with attaching map given by the restriction of $d c$ (and hence of $d$ ) to $X_{m-l, 1}^{n} \circ X_{l, 1}^{n-1}$.

(ii) $X_{m, l}^{n}$ is homeomorphic to the adjunction space $X_{m, l+1}^{n} \cup C X_{m-l, 1}^{n} \times X_{l, l}^{n}$ with attaching map given by the restriction of $\beta(\alpha \times \mathrm{id})$ (and hence of $\beta$ ) to $X_{m-l, 1}^{n} \times X_{l, l}^{n}$.

As a consequence of Lemma 2.4 we have 
LEMMA 2.5. Let $1 \leqq l<m$. Then

(i) $X_{m, l}^{n} /\left(X_{m, l+1}^{n} \cup X_{m-1, l-1}^{n}\right)$ and $E X_{m-l, 1}^{n} \wedge E X_{l, 1}^{n-1}$ are homeomorphic.

(ii) $X_{m, l}^{n} / X_{m, l+1}^{n}$ and $E X_{m-l, 1}^{n} \vee E\left(X_{m-l, 1}^{n} \wedge X_{l, l}^{n}\right)$ have the same homotopy type.

(iii) $X_{m, l}^{n} / X_{m-1, l-1}^{n}$ is homeomorphic to the adjunction space $X_{m, l+1}^{n} / X_{m-1, l}^{n}$ $\cup C\left(X_{m-l, 1}^{n} \circ X_{l, 1}^{n-1}\right)$ with attaching map given by the composition of the attaching map in 2.4(i) and the obvious collapsing map.

Proof. (i) follows directly from Lemma 2.4(i) and the easy observation that $(C(X \circ Y), X \circ Y) \cong(C X \times C Y, X \circ Y)$ and $(E X \times E Y, E X \vee E Y)$ are relatively homeomorphic. Similarly (ii) comes from Lemma 2.4(ii) and the facts (1) that $(C X \times Y, X \times Y)$ and $(E X \times Y$, point $\times Y)$ are relatively homeomorphic and (2) that the quotient $E X \times Y /$ point $\times Y$ has the same homotopy type as $E X \vee E(X \wedge Y)$. Finally (iii) follows from Lemma 2.4(i) and the simple observation that $X_{m, l+1}^{n}$ $\cap X_{m-1, l-1}^{n}=X_{m-1, l}^{n}$.

With these preliminaries over we can proceed to consider the desuspension properties of the above and related spaces. Our approach will be to "desuspend up to homotopy equivalence" certain of the above attaching maps.

First we recall some elementary homotopy theory. Suppose $X$ and $X^{\prime}$ are 1-connected and $A$ and $A^{\prime}$ are 0 -connected finite $\mathrm{CW}$ complexes and

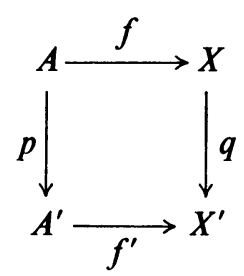

is a commutative diagram of maps with $p$ and $q$ homotopy equivalences. Then the adjunction spaces $X \cup, C A$ and $X^{\prime} \cup_{f^{\prime}} C A^{\prime}$ have the same homotopy type. An explicit homotopy equivalence

$$
F: X \cup_{f} C A \rightarrow X^{\prime} \cup_{f^{\prime}} C A^{\prime}
$$

is given by $F \mid X=q$ and $F \mid C A=C(p)$ the conal extension of $p$. We call $F$ the conal homotopy equivalence determined by $p$ and $q$. Our interest lies in the situation $A^{\prime}=E A^{\prime \prime}, X^{\prime}=E X^{\prime \prime}$ suspension spaces and $f^{\prime}=E f^{\prime \prime}$ a suspension map. Then $X \cup_{f} C A$ will have the homotopy type of the suspension space $E X^{\prime \prime} \cup_{E f^{\prime \prime}} C\left(E A^{\prime \prime}\right)$ $\cong E\left(X^{\prime \prime} \cup_{f^{\prime \prime}} C A^{\prime \prime}\right)$.

We now apply these remarks to the space $X_{m, m-1}^{n} / X_{m-1, m-2}^{n}$. According to Lemma 2.5(iii) the attaching map in the adjunction space

$$
X_{m, m}^{n} / X_{m-1, m-1}^{n} \cup C\left(X_{1,1}^{n} \circ X_{m-1,1}^{n-1}\right)
$$

is the composite

$$
X_{1,1}^{n} \circ X_{m-1,1}^{n-1} \rightarrow X_{m, m}^{n} \cup X_{m-1, m-2}^{n} \rightarrow \frac{X_{m, m}^{n} \cup X_{m-1, m-2}^{n}}{X_{m-1, m-2}^{n}} \cong \frac{X_{m, m}^{n}}{X_{m-1, m-1}^{n}} .
$$


The first map on $X_{1,1}^{n} \times C X_{m-1,1}^{n-1}$ is $\beta \circ\left(\mathrm{id} \times\left(h_{S(m-1)} \circ \alpha\right)\right)$ and on $C X_{1,1}^{n} \times X_{m-1,1}^{n-1}$ is $\beta \circ\left(\alpha \times h_{S(m-1)}\right)$ where $\beta: X_{1,0}^{n} \times X_{m-2, m-2}^{n} \rightarrow X_{m-1, m-2}^{n}$ is the shuffle map. A more familiar description of this attaching map via the identifications $X_{1,1}^{n}=S^{n-1}$, $X_{m, m}^{n} / X_{m-1, m-1}^{n}=S P^{m} S^{n-1} / S P^{m-1} S^{n-1}$ is given by

$$
\begin{aligned}
S^{n-1} \times C X_{m-1,1}^{n-1} \rightarrow S^{n-1} \times S P^{m-1} S^{n-1} & \rightarrow S P^{m} S^{n-1} \rightarrow S P^{m} S^{n-1} / S P^{m-1} S^{n-1}, \\
C S^{n-1} \times X_{m-1,1}^{n-1} & \rightarrow \text { basepoint. }
\end{aligned}
$$

As point $\times C X_{m-1,1}^{n-1}$ is also mapped to the basepoint, this attaching map factors as

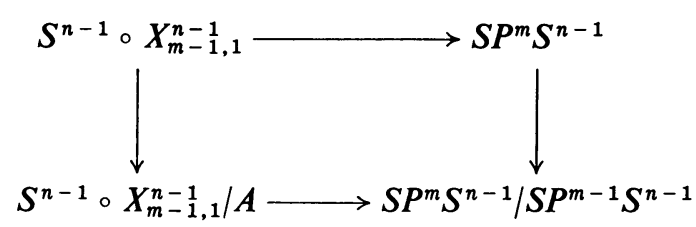

where $A=$ point $\times C X_{m-1,1}^{n-1} \cup C S^{n-1} \times X_{m-1,1}^{n-1}$ is contractible (in itself). Thus the collapsing map $p$ is a homotopy equivalence. Moreover, setting $S=S^{n-1}$ and $X=X_{m-1,1}^{n-1}$ we can impose a suspension structure on $S \circ X / A$ by the following (obvious) homeomorphisms:

$$
\begin{aligned}
\frac{S \circ X}{A} & \cong \frac{S \times C X}{S \times X \cup \operatorname{point} \times C X} \cong \frac{C S^{n-2} \times C X}{C S^{n-2} \times X \cup S^{n-2} \times C X} \\
& \cong \frac{C\left(S^{n-2} \circ X\right)}{S^{n-2} \circ X}=E\left(S^{n-2} \circ X\right)
\end{aligned}
$$

But $X_{1,0}^{n-1} \times X_{m-1,1}^{n-1} \cong C S^{n-2} \times C X$ and so the map inducing the lower horizontal map of (5) is obviously the shuffle map. Thus we have proven

Proposition 2.6. $X_{m, m-1}^{n} / X_{m-1, m-2}^{n}$ has the homotopy type of the suspension space

$$
E X_{m, 1}^{n-1} \cup_{E \psi} C\left(E\left(S^{n-2} \circ X_{m-1,1}^{n-1}\right)\right)
$$

where $\psi$ is the map defined in (4), i.e. $\psi$ is given by

$$
\begin{aligned}
& S^{n-2} \times C X_{m-1,1}^{n-1} \stackrel{\mathrm{id} \times \alpha}{\longrightarrow} X_{1,1}^{n-1} \times X_{m-1,0}^{n-1} \stackrel{\beta}{\longrightarrow} X_{m, 1}^{n-1}, \\
& C S^{n-2} \times X_{m-1,1}^{n-1} \stackrel{\alpha \times \mathrm{id}}{\longrightarrow} X_{1,0}^{n-1} \times X_{m-1,1}^{n-1} \stackrel{\beta}{\longrightarrow} X_{m, 1}^{n-1} .
\end{aligned}
$$

Moreover an explicit homotopy equivalence is given by the composition $F \circ G$, $G$ the homeomorphism of Lemma 2.5 (iii) and $F$ the conal homotopy equivalence determined by the collapsing map $p$

$$
p: S^{n-1} \circ X_{m-1,1}^{n-1} \rightarrow \frac{S^{n-1} \circ X_{m-1,1}^{n-1}}{A}
$$

and the homeomorphism $q: X_{m, m}^{n-1} / X_{m-1, m-1}^{n-1} \cong E X_{m, 1}^{n-1}$.

The particular case $m=2$ yields a slightly stronger assertion: 
COROLlaRY 2.7. $X_{2,1}^{n}$ has the homotopy type of the suspension space

$$
E X_{2,1}^{n-1} \cup_{E \psi} C\left(E\left(S^{n-2} \circ S^{n-2}\right)\right)
$$

and an explicit homotopy equivalence is given by $F \circ G \circ p$ where $F, G, \psi$ are as in 2.6 and $p: X_{2,1}^{n} \rightarrow X_{2,1}^{n} / X_{1,0}^{n}$ is the collapsing map (a homotopy equivalence).

We now restrict our attention to the case $m=3$. We wish to prove

Proposition 2.8. $X_{3,2}^{n} / X_{2,1}^{n}$ has the homotopy type of a double suspension space.

An important step for this is

Proposition 2.9. $X_{3,1}^{n-1}$ has the homotopy type of a suspension.

Proof (of 2.9). By Lemma 2.4(i) $X_{3,1}^{n-1}$ is homeomorphic to the adjunction space

$$
\left(X_{3,2}^{n-1} \cup X_{2,0}^{n-1}\right) \cup_{\phi} C\left(X_{2,0}^{n-1} \circ S^{n-3}\right) .
$$

Since $X_{2,0}^{n-1} \cong C X_{2,1}^{n-1}$ the collapsing map $X_{3,2}^{n-1} \cup X_{2,0}^{n-1} \rightarrow X_{3,2}^{n-1} / X_{2,1}^{n-1}$ is a homotopy equivalence and so the adjunction space (7) is homotopy equivalent to the adjunction space

$$
X_{3,2}^{n-1} / X_{2,1}^{n-1} \cup_{\varphi} C\left(X_{2,1}^{n-1} \circ S^{n-3}\right) .
$$

Here the attaching map $\varphi$ is given by

$$
\begin{gathered}
X_{2,1}^{n-1} \times C S^{n-3} \rightarrow X_{2,1}^{n} \times X_{1,1}^{n-1} \rightarrow X_{3,2}^{n-1} \rightarrow X_{3,2}^{n-1} / X_{2,1}^{n-1}, \\
A=\text { point } \times C S^{n-3} \cup C X_{2,1}^{n-1} \times S^{n-3} \rightarrow \text { basepoint, }
\end{gathered}
$$

and so we again have a factorization

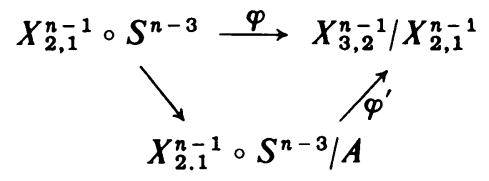

Recall the homeomorphism $\left(X_{2,1}^{n-1} \circ S^{n-3}\right) / A \cong\left(X_{2,1}^{n-1} \times C S^{n-3}\right) / A^{\prime}$, where $A^{\prime}=X_{2,1}^{n-1} \times S^{n-3} \cup$ point $\times C S^{n-3}$, and the homotopy equivalence

$$
\frac{X_{2,1}^{n-1} \times C S^{n-3}}{A^{\prime}} \rightarrow Z=\frac{\left\{S P^{2} S^{n-2} / S^{n-2} \cup_{\varphi} C\left(S^{n-2} \circ S^{n-2}\right)\right\} \times C S^{n-3}}{A^{\prime \prime}}
$$

where

$$
A^{\prime \prime}=\left\{S P^{2} S^{n-2} / S^{n-2} \cup_{\varphi} C\left(S^{n-2} \circ S^{n-3}\right)\right\} \times S^{n-3} \cup \text { point } \times C S^{n-3} .
$$

From this and the homeomorphisms

$$
\begin{aligned}
S P^{2} S^{n-2} / S^{n-2} & \cong C X_{2,1}^{n-2} / X_{2,1}^{n-2}=E X_{2,1}^{n-2}, \\
\frac{E X_{2,1}^{n-2} \times C S^{n-3}}{E X_{2,1}^{n-2} \times S^{n-3} \cup \text { point } \times C S^{n-3}} & \cong \frac{C X_{2,1}^{n-2} \times C S^{n-3}}{X_{2,1}^{n-2} \circ S^{n-3}} \\
& \cong \frac{C\left(X_{2,1}^{n-2} \circ S^{n-3}\right)}{X_{2,1}^{n-2} \circ S^{n-3}}=E\left(X_{2,1}^{n-2} \circ S^{n-3}\right)
\end{aligned}
$$


we obtain a homeomorphism

$$
Z \rightarrow E\left(X_{2,1}^{n-2} \circ S^{n-3}\right) \cup_{y} C\left(S^{n-2} \circ S^{n-3}\right) \times C S^{n-3}
$$

where the attaching map $\gamma$ is given by

$$
\begin{aligned}
\left(S^{n-2} \circ S^{n-3}\right) \times C S^{n-3} & \rightarrow \frac{S P^{2} S^{n-3}}{S^{n-2}} \times C S^{n-3} \rightarrow \frac{\left(S P^{2} S^{n-2} / S^{n-2}\right) \times C S^{n-3}}{A} \\
& \cong E\left(X_{2,1}^{n-2} \circ S^{n-3}\right), \\
& C\left(S^{n-2} \circ S^{n-3}\right) \times S^{n-3} \rightarrow \text { basepoint. }
\end{aligned}
$$

Since the map $\varphi$ sends $D^{n-1} \times C S^{n-3}$ to the basepoint we obtain induced maps $\varphi^{\prime \prime}, \varphi^{\prime \prime}, \varphi^{(1 v)}$ and the commutative diagram

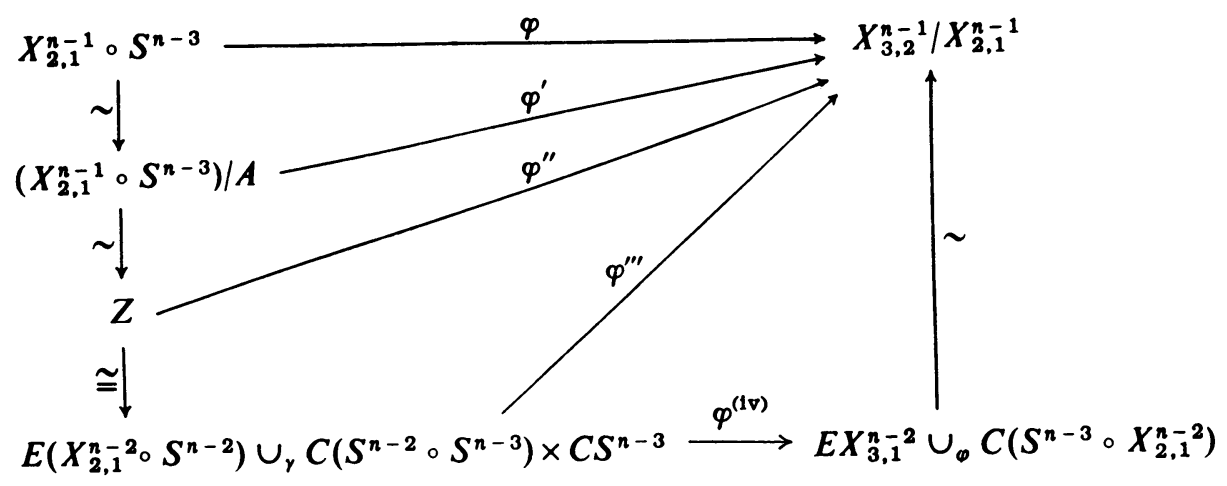

The end is now in sight. To complete the proof of 2.9 we need only show that $\varphi^{(i v)}$ is homotopy equivalent to a suspension. First note that $\varphi^{(\mathrm{iv})} \mid E\left(X_{2,1}^{n-2} \circ S^{n-3}\right)$ is already a suspension map since it is induced by the "shuffle" map

$$
C X_{2,1}^{n-2} \times C S^{n-3} \rightarrow C X_{3,1}^{n-2} \text {. }
$$

Furthermore $\varphi^{(\mathrm{iv})}$ is induced by the composite $h_{4} h_{3} h_{2} h_{1}$ of homeomorphisms

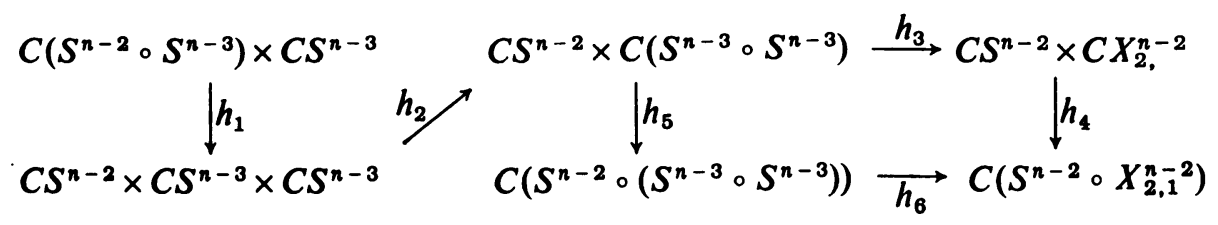

Now there exists a homeomorphism $h_{7}$

$$
\begin{aligned}
E\left(X_{2,1}^{n-2} \circ S^{n-3}\right) \cup y^{\prime} C\left(S^{n-2} \circ\right. & \left.\left(S^{n-3} \circ S^{n-3}\right)\right) \\
& \stackrel{h_{7}}{\longrightarrow} E\left(X_{2,1}^{n-2} \circ S^{n-3}\right) \cup_{y} C\left(S^{n-2} \circ S^{n-3}\right) \times C S^{n-3}
\end{aligned}
$$

which is the identity on $E\left(X_{2,1}^{n-2} \circ S^{n-3}\right)$ and $\left(h_{5} h_{2} h_{1}\right)^{-1}$ on

$$
C\left(S^{n-2} \circ\left(S^{n-3} \circ S^{n-3}\right)\right) \text {. }
$$


Here $\gamma^{\prime}=\gamma\left(h_{5} h_{2} h_{1}\right)^{-1} \mid\left(S^{n-2} \circ\left(S^{n-3} \circ S^{n-3}\right)\right) . \varphi^{(1 v)} h_{7}$ is now induced by $h_{6}$. The attaching map $\varphi$ factors as

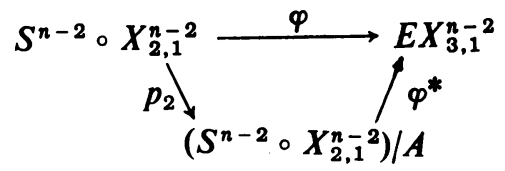

where $A=$ point $\times C X_{2,1}^{n-2} \cup C S^{n-2} \times X_{2,1}^{n-2} \cdot p_{2}$ is a homotopy equivalence and $\varphi^{*}$ becomes $E \psi$ when $\left(S^{n-2} \circ X_{2,1}^{n-2}\right) / A$ is identified as $E\left(S^{n-3} \circ X_{2,1}^{n-2}\right)$.

The attaching map $\gamma^{\prime}$ also factors as

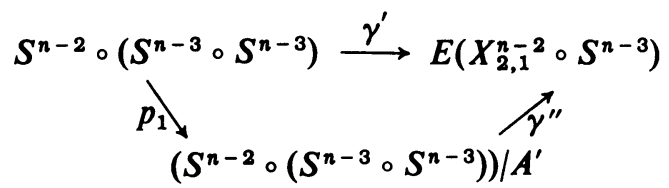

where $A^{\prime}=$ point $\times C\left(S^{n-3} \circ S^{n-3}\right) \cup C S^{n-3} \times\left(S^{n-3} \circ S^{n-3}\right)$ and $p_{1}$ is a homotopy equivalence. Thus we have a commutative diagram with $\varphi^{(v)}$ induced by $\varphi^{(1 v)}$

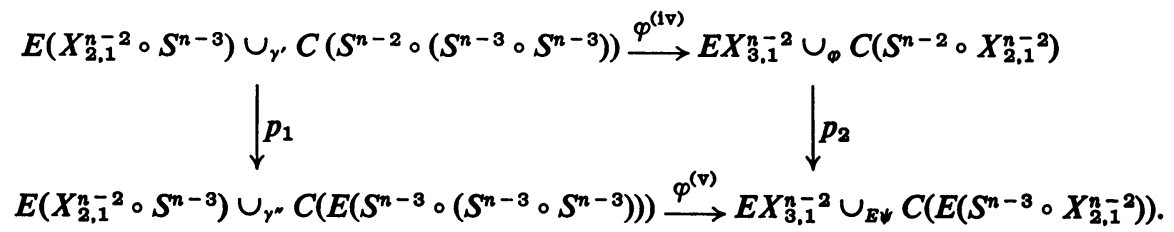

But $\varphi^{(\mathrm{v})}$ restricts to a suspension map $E\left(X_{2,1}^{n-2} \circ S^{n-3}\right) \rightarrow E X_{3,1}^{n-2}$ and moreover maps the suspension variable of $C\left(E\left(S^{n-3} \circ\left(S^{n-3} \circ S^{n-3}\right)\right)\right)$ linearly onto the suspension variable of $C\left(E\left(S^{n-3} \circ X_{2,1}^{n-2}\right)\right)$. Thus $\varphi^{(v)}$ will be a suspension map (and $X_{3,1}^{n-1}$ homotopy equivalent to a suspension space) if the attaching map $\gamma^{\prime \prime}$ is also a suspension map. But this is a simple matter of direct verification (or is obvious by construction!).

We need examine only how $\gamma^{\prime}$ behaves on $S^{n-2} \times C\left(S^{n-3} \circ S^{n-3}\right)$ since $\gamma^{\prime}$ sends point $\times C\left(S^{n-3} \circ S^{n-3}\right) \cup C S^{n-2} \times\left(S^{n-3} \circ S^{n-3}\right)$ to the basepoint (south pole). Recall that the suspension structure of $E\left(S^{n-3} \circ\left(S^{n-3} \circ S^{n-3}\right)\right)$ comes from $\left(S^{n-2} \times C\left(S^{n-3} \circ S^{n-3}\right)\right) / A^{\prime \prime}$,

$$
A^{\prime \prime}=\text { point } \times C\left(S^{n-3} \circ S^{n-3}\right) \cup C S^{n-2} \times\left(S^{n-3} \circ S^{n-3}\right)
$$

via

$$
\frac{C S^{n-3} \times C\left(S^{n-3} \circ S^{n-3}\right)}{S^{n-3} \circ\left(S^{n-3} \circ S^{n-3}\right)} \cong \frac{C\left(S^{n-3} \circ\left(S^{n-3} \circ S^{n-3}\right)\right)}{S^{n-3} \circ\left(S^{n-3} \circ S^{n-3}\right)} .
$$

Similarly that of $E\left(X_{2,1}^{n-2} \circ S^{n-3}\right)$ comes from

$$
\frac{C X_{2,1}^{n-2} \times C S^{n-3}}{X_{2,1}^{n-2} \circ S^{n-3}} \cong \frac{C\left(X_{2,1}^{n-2} \circ S^{n-3}\right)}{X_{2,1}^{n-2} \circ S^{n-3}}
$$


Let $\left(x_{2},\left[x_{3}, t_{3}\right]\right) \in S^{n-3} \times C S^{n-3} \subset S^{n-3} \circ S^{n-3}$ so that

$$
a=\left(\left[x_{1}, t_{1}\right],\left[\left(x_{2},\left[x_{3}, t_{3}\right]\right), u\right] \in C S^{n-3} \times C\left(S^{n-3} \circ S^{n-3}\right) .\right.
$$

Then $a$ is mapped to $b=\left(\left[\left[x_{1}, t_{1}\right],\left[x_{2}, u\right]\right],\left[x_{3}, t u\right]\right) \in X_{2,0}^{n-2} \times C S^{n-3}$. As an element of $E\left(S^{n-3} \circ\left(S^{n-3} \circ S^{n-3}\right)\right) a$ has $t_{1}$ or $u$ as suspension variable according as $u \leqq t_{1}$ or $t_{1} \leqq u$. As an element of $C X_{2,1}^{n-2} \times C S^{n-3} b$ has the description

$$
\begin{aligned}
& b=\left(\left[\left(x_{1},\left[x_{2}, u / t_{1}\right]\right), t_{1}\right],\left[x_{1}, t_{3} u\right]\right) \text { if } u \leqq t_{1}, \\
& =\left(\left[\left(\left[x_{1}, t_{1} / u\right], x_{2}\right), u\right],\left[x_{3}, t_{3} u\right]\right) \quad \text { if } t_{1}>u \text {. }
\end{aligned}
$$

Hence the suspension variable of $b$ is $t_{1}$ or $u$ according as $t_{3} u \leqq t_{1}$ or $t_{1} \leqq u$. So the condition $u \leqq t_{1}$ implies $t_{3} u \leqq t_{1}$ in which case $t_{1}$ is the suspension variable for both $a$ and $b$. The other alternative $t_{1} \leqq u$ makes $u$ the common suspension variable.

On the other hand let $\left(\left[x_{2}, t_{2}\right], x_{3}\right) \in C S^{n-3} \times S^{n-3}$ so that

$$
a=\left(\left[x_{1}, t_{1}\right],\left[\left(\left[x_{2}, t_{2}\right], x_{3}\right), u\right]\right) \in C S^{n-3} \times C\left(S^{n-3} \circ S^{n-3}\right) .
$$

Then $a$ is mapped to $b=\left(\left[\left[x_{1}, t_{1}\right],\left[x_{2}, t_{2} u\right]\right],\left[x_{3}, u\right]\right) \in X_{2,0}^{n-2} \times C S^{n-3}$. As an element of $C X_{2,1}^{n-2} \times C S^{n-3} b$ looks like

$$
\begin{aligned}
& b=\left(\left[\left(x_{1},\left[x_{2}, t_{2} u / t_{1}\right]\right), t_{1}\right],\left[x_{3}, u\right]\right) \quad \text { if } t_{2} u \leqq t_{1}, \\
& =\left(\left[\left(\left[x_{1}, t_{1} / t_{2} u\right], x_{2}\right), t_{2} u\right],\left[x_{3}, u\right]\right) \text { if } t_{1} \leqq t_{2} u \text {. }
\end{aligned}
$$

Now if $u \leqq t_{1}$ then $a$ has suspension variable $t_{1}$. But then so does $b$. If $t_{1} \leqq u$ then $a$ has suspension variable $u$. So does $b$ but this requires a little checking: if $t_{2} u \leqq t_{1}$ then $b$ has suspension variable $u$ from the top half of (8), and if $t_{1} \leqq t_{2} u$ then $b$ has suspension variable $u$ from the bottom half of (8).

Hence $\gamma^{\prime \prime}$ is a suspension map and the proof of Proposition 2.9 is complete.

The proof of 2.8 is based on the following elementary observation, the proof of which is left to the reader: suppose $f: X \circ Y \rightarrow Z$ is a map which factors as $f^{\prime} p=f$

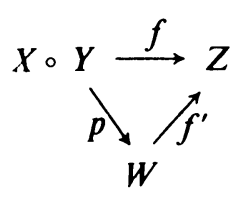

where $p$ collapses $X \times$ vertex $_{C Y}$ to a point and collapses vertex ${ }_{C X} \times Y$ to a (different) point and $W$ is the resulting quotient space (easy to see that $W \cong E(X \times Y)$ with these collapsed points corresponding to the north and south poles). Then $f$ is homotopic to the map $g$ given by

$$
\begin{array}{rlrl}
g(x,[y, t]) & =f(x,[y, 2 t]), & & 0 \leqq t \leqq \frac{1}{2}, \\
& =f(x,[y,-2 t+2]), & \frac{1}{2} \leqq t \leqq 1 ; \\
g(C X \times Y) & =\text { basepoint. } & &
\end{array}
$$


By Proposition 2.6 there is a homotopy equivalence

$$
X_{3,2}^{n} / X_{2,1}^{n} \rightarrow E X_{3,1}^{n-1} \cup_{E \downarrow} C\left(E\left(S^{n-2} \circ X_{2,1}^{n-1}\right)\right) .
$$

From the above observation the map $\psi: S^{n-2} \circ X_{2,1}^{n-1} \rightarrow X_{3,1}^{n-1}$ is homotopic to the map $\psi^{\prime}: S^{n-2} \circ X_{2,1}^{n-1} \rightarrow X_{3,1}^{n-1}$ defined above. Thus we have a commutative diagram

$$
\begin{aligned}
& S^{n-2} \circ X_{2,1}^{n-1} \stackrel{\psi^{\prime}}{\longrightarrow} X_{3,1}^{n-1} \longrightarrow X_{3,2}^{n-1} / X_{2,1}^{n-1} \cup C\left(X_{2,1}^{n-1} \circ S^{n-3}\right) \\
& p \searrow / f^{\prime} \\
& \left(S^{n-2} \circ X_{2,1}^{n-1}\right) / A
\end{aligned}
$$

where $A=$ point $\times C X_{2,1}^{n-1} \cup C S^{n-2} \times X_{2,1}^{n-1}$. Set

$$
\begin{aligned}
\bar{X} & =\left\{\left[x, \frac{1}{2}\right] \in C X_{2,1}^{n-1} \mid x \in X_{2,1}^{n-1}\right\}, \\
C_{+} X & =\left\{[x, t] \in C X_{2,1}^{n-1} \mid x \in X_{2,1}^{n-1}, 0 \leqq t \leqq \frac{1}{2}\right\}, \\
C_{-} X & =\left\{[x, t] \in C X_{2,1}^{n-1} \mid x \in X_{2,1}^{n-1}, \frac{1}{2} \leqq t \leqq 1\right\} .
\end{aligned}
$$

From the definition of $\psi^{\prime}$ we have that the $f^{\prime}$-pre-image of $X_{3,2}^{n-1} / X_{2,1}^{n-1}$ is the $p$ image of $S^{n-2} \times \bar{X} \cup C S \times X_{2,1}^{n-1} \cup \mathrm{pt} \times C X_{2,1}^{n-1}$.

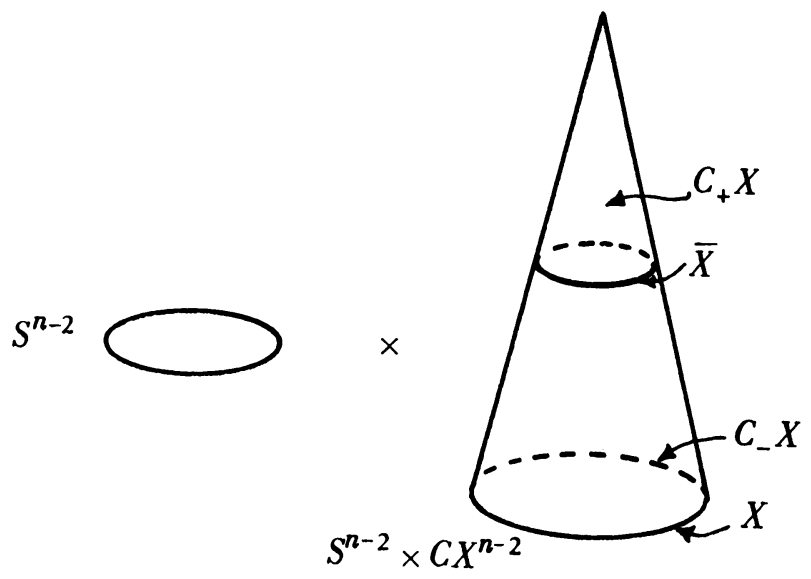

We define maps

(i) $C S^{n-3} \times C_{+} X \rightarrow S^{n-2} \times C_{+} X$,

(ii) $C^{n-3} \times C_{-} X \rightarrow S^{n-2} \times C_{-} X$,

via the maps $C S^{n-3} \cong D^{n-2} \stackrel{h}{\rightarrow} S^{n-2}$ and $C_{ \pm} X \stackrel{\text { ld }}{\rightarrow} C_{ \pm} X$. In (i) the boundary $S^{n-3} \times C_{+} X \cup C S^{n-3} \times X$ is mapped onto point $\times C_{+} X \cup S^{n-2} \times \bar{X}$ and in (ii) the boundary

$$
S^{n-2} \times C_{-} X \cup C S^{n-3} \times X \text { onto pt } \times C_{-} X \cup S^{n-2} \times(\bar{X} \cup X) .
$$

Consequently there exists a homeomorphism

$$
Y \cong\left(S^{n-2} \times \bar{X} \cup \text { point } \times C X \cup C S^{n-2} \times X\right) \cup C S^{n-3} \times C_{+} X \cup C S^{n-3} \times C_{-} X
$$


and so a homeomorphism

$$
\frac{Y}{A} \cong Z_{1}=\frac{S^{n-2} \times \bar{X}}{\text { point } \times X} \cup_{\alpha_{1}} C S^{n-3} \times C_{+} X \cup_{\alpha_{2}} C S^{n-3} \times C_{-} X
$$

with attaching maps $\alpha_{1}$ and $\alpha_{2}$ derived from (i) and (ii) above together with the collapsing map $Y \rightarrow Y / A$.

Now the collapsing map

$$
\frac{S^{n-2} \times \bar{X}}{\text { point } \times \bar{X}} \rightarrow \frac{S^{n-2} \times \bar{X}}{S^{n-2} \vee \bar{X}}
$$

induces a collapsing map

$$
Z_{1} \rightarrow Z_{2}=\left(\frac{S^{n-2} \times \bar{X}}{S^{n-2} \vee \bar{X}}\right) \cup_{\alpha_{1}^{\prime}} C S^{n-3} \times C_{+} X \cup_{\alpha_{2}^{\prime}} C S^{n-3} \times C_{-} X
$$

But

$$
\frac{S^{n-2} \times \bar{X}}{\text { point } \times \bar{X}} \sim S^{n-2} \vee\left(S^{n-2} \wedge \bar{X}\right)
$$

and so the map (10) is obviously homotopy equivalent to a suspension map. And it follows easily that the map (11) is also homotopy equivalent to a suspension map. As the $\operatorname{map} f^{\prime}$ of (9) factors as

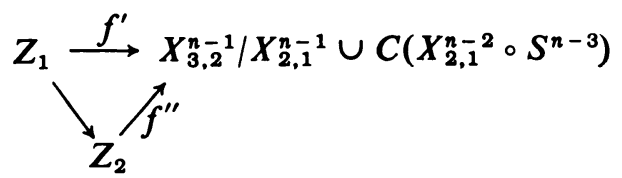

we see that to prove $f^{\prime}$ is homotopy equivalent to a suspension, it suffices to prove that $f^{\prime \prime}$ is. Note that

$$
\left(f^{\prime \prime}\right)^{-1}\left(\frac{X_{3,2}^{n-1}}{X_{2,1}^{n-1}}\right)=\frac{S^{n-2} \times \bar{X}}{S^{n-2} \vee \bar{X}}
$$

The rest of the proof consists of two steps.

Step 1. $f^{\prime \prime} \mid\left(S^{n-2} \times \bar{X}\right) /\left(S^{n-2} \vee \bar{X}\right)$ is homotopy equivalent to a suspension.

Recall the homotopy equivalence

$$
\bar{X}\left(=X_{2,1}^{n-1}\right) \rightarrow E X_{2,1}^{n-2} \cup C\left(S^{n-2} \circ S^{n-3}\right)
$$

from which we obtain a homotopy equivalence

$$
\frac{S^{n-2} \times \bar{X}}{S^{n-2} \vee \bar{X}} \rightarrow Z_{3}=\frac{S^{n-2} \times\left(E X_{2,1}^{n-2} \cup C\left(S^{n-2} \circ S^{n-3}\right)\right)}{S^{n-2} \vee\left(E X_{2,1}^{n-2} \cup C\left(S^{n-2} \circ S^{n-3}\right)\right)}
$$

and a factoring 


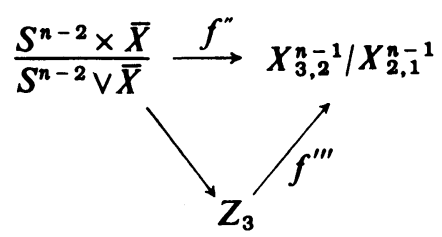

In an obvious way $Z_{3}$ is homeomorphic to the space

$$
Z_{4}=\left(\frac{S^{n-2} \times E X_{2,1}^{n-2}}{S^{n-2} \vee E X_{2,1}^{n-2}}\right) \cup_{\delta} C S^{n-3} \times C\left(S^{n-2} \circ S^{n-3}\right)
$$

where $\delta$ is given by

$$
\begin{aligned}
& S^{n-3} \times C\left(S^{n-2} \circ S^{n-3}\right) \rightarrow \text { basepoint, } \\
& C S^{n-3} \times S^{n-2} \times C S^{n-3} \rightarrow S^{n-2} \times E X_{2,1}^{n-2}, \\
& C S^{n-3} \times C S^{n-2} \times S^{n-3} \rightarrow \text { basepoint. }
\end{aligned}
$$

With the inverse of the above homeomorphism $Z_{3} \rightarrow Z_{4}$ the map $f^{m}$ defines a map $f^{(\text {iv) }}: Z_{4} \rightarrow X_{3,2}^{n-1} / X_{2,1}^{n-1}$. Checking the above definitions we see that $f^{(\text {(v) }}$ is induced by the composite map

$$
\begin{aligned}
C S^{n-3} \times C\left(S^{n-2} \circ S^{n-3}\right) & \rightarrow C S^{n-3} \times C S^{n-2} \times C S^{n-3} \rightarrow C S^{n-2} \times C\left(S^{n-3} \circ S^{n-3}\right) \\
& \rightarrow C S^{n-2} \times C X_{2,1}^{n-2} \rightarrow C\left(S^{n-2} \circ X_{2,1}^{n-2}\right) .
\end{aligned}
$$

Therefore if we replace $Z_{4}$ by the homeomorphic (to $Z_{4}$ )

$$
Z_{5}=\frac{C S^{n-3} \times C X_{2,1}^{n-2}}{S^{n-3} \circ X_{2,1}^{n-2}} \cup C S^{n-2} \times C\left(S^{n-3} \circ S^{n-3}\right),
$$

the resulting map $Z_{5} \rightarrow E X_{3,1}^{n-2} \cup C\left(S^{n-2} \circ X_{2,1}^{n-2}\right)$ is induced by the composite

$$
C S^{n-2} \times C\left(S^{n-3} \circ S^{n-3}\right) \rightarrow C S^{n-2} \times C X_{2,1}^{n-2} \rightarrow C\left(S^{n-2} \circ X_{2,1}^{n-2}\right)
$$

and so restricts to a suspension map $E\left(S^{n-3} \circ X_{2,1}^{n-2}\right) \rightarrow E X_{3,1}^{n-2}$, and on

$$
C\left(S^{n-2} \circ\left(S^{n-3} \circ S^{n-3}\right)\right)
$$

is the conal extension of the map

$$
S^{n-2} \circ\left(S^{n-3} \circ S^{n-3}\right) \stackrel{\text { id } \circ \psi}{\longrightarrow} S^{n-2} \circ X_{2,1}^{n-2} .
$$

Hence via the usual homotopy equivalences we obtain a commutative diagram

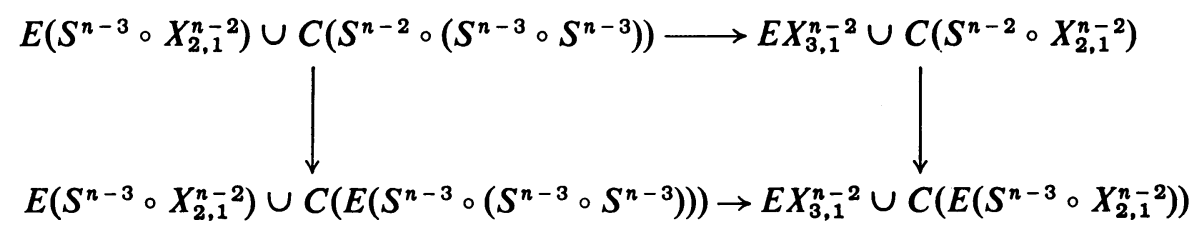

where the bottom horizontal map is a suspension map and the vertical maps 
homotopy equivalences. Thus $f^{\prime \prime} \mid\left(S^{n-2} \times \bar{X}\right) /\left(S^{n-2} \vee \bar{X}\right)$ is homotopy equivalent to a suspension.

Step 2. We have constructed a commutative diagram

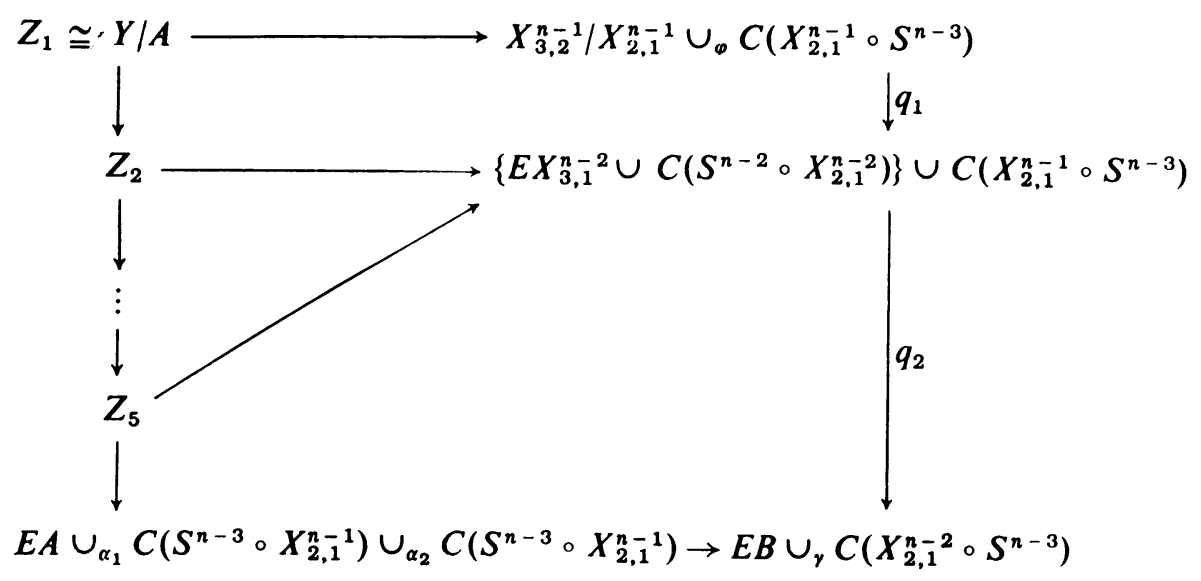

where the bottom horizontal map restricted to $E A$ is a suspension map $\tilde{h}: E A \rightarrow E B$,

$$
E A=E\left(S^{n-3} \circ X_{2,1}^{n-2}\right) \cup C\left(E\left(S^{n-3} \circ\left(S^{n-3} \circ S^{n-3}\right)\right)\right)
$$

and

$$
E B=E X_{3,1}^{n-2} \cup C\left(E\left(S^{n-3} \circ X_{2,1}^{n-2}\right)\right) .
$$

The attaching maps satisfy the relation $\tilde{h} \circ \alpha_{i}=\gamma \circ T$ where $T: S^{n-3} \circ X_{2,1}^{n-1} \rightarrow$ $X_{2,1}^{n-1} \circ S^{n-3}$ is the switch homeomorphism. As

$$
\alpha_{i}\left(S^{n-3} \times C X_{2,1}^{n-1} \cup C S^{n-3} \times \text { point }\right)=\text { basepoint } \quad(i=1 \text { and } 2)
$$

and

$$
\gamma\left(C X_{2,1}^{n-1} \times S^{n-3} \cup \text { point } \times C S^{n-3}\right)=\text { basepoint, }
$$

we will be done if $X_{2,1}^{n-1}$ is a suspension space $X_{2,1}^{n-1}=E Y$, for then we have a commutative diagram

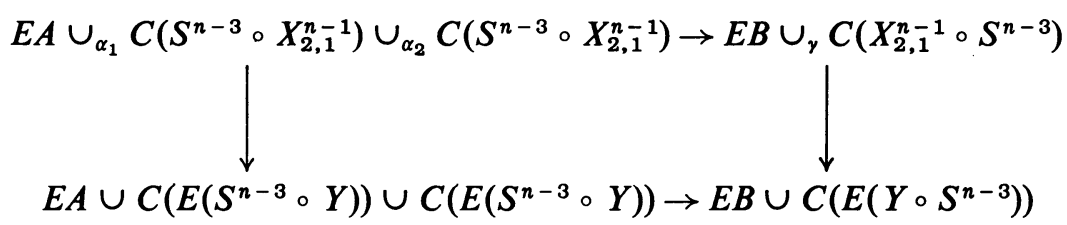

with the bottom horizontal map a suspension. And in fact by Lemma 2.1 of [1] $X_{2,1}^{n-1}$ is a suspension. However we can also give a self-contained proof using the homotopy equivalence $X_{2,1}^{n-1} \rightarrow E Y^{\prime}$ of Corollary 2.7. Since the attaching maps $\alpha_{1}, \alpha_{2}($ and $\gamma)$ send $\left(S^{n-2} \circ X_{1,0}^{n}\right)\left(X_{1,0}^{n} \circ S^{n-3}\right)$ to the basepoint, we obtain a new diagram similar to (12) with $E Y^{\prime}$ and $Y^{\prime}$ replacing $X_{2,1}^{n-1}$ and $Y$ respectively. The conclusion again is that the bottom horizontal map is a suspension. Whence $X_{3,2}^{n} / X_{2,1}^{n}$ has the homotopy type of a double suspension. 
REMARKS. 1. For $m=2$ the preceding argument would suffice to prove that $X_{2,1}^{n} / X_{1,0}^{n}$ (and hence $X_{2,1}^{n}$ itself) has the homotopy type of a double suspension. However Lemma 2.1 of [1] asserts much more: $X_{2,1}^{n}$ is homeomorphic to the join $S^{n-1} \circ P^{n-1}$ of $S^{n-1}$ with real projective $n$-space $P^{n-1}$. The subsets of $X_{2,1}^{n}$ corresponding to the "ends" of this join are $S^{n-1} \cong\left\{[x, x] \in X_{2,1}^{n} \mid x \in S^{n-1}\right\}$ and $P^{n-1} \cong\left\{[x,-x] \in X_{2,1}^{n} \mid x \in S^{n-1}\right\}$. It seems reasonable to conjecture that either $X_{m, m-1}^{n}$ or $X_{m, m-1}^{n} / X_{m-1, m-2}^{n}$ is homeomorphic to a join of the form $S^{n-1} \circ Z$ for some subcomplex $Z$ (the problem is to determine $Z$ ).

2. Of immediate concern for constructing nontrivial maps $S P^{m} S^{n} \rightarrow S^{n}$ would be the result that $X_{m, m-1}^{n} / X_{m-1, m-2}^{n}$ has the homotopy type of a double suspension for all $m$ (not just $m=2$ or 3). The techniques of this paragraph seem suitable for this. What is needed is (a) $X_{m, 1}^{n-1}$ has the homotopy type of a suspension, and (b) the "shuffle" map $S^{n-2} \circ X_{m-1,1}^{n-1} \rightarrow X_{m, 1}^{n-1}$ is homotopy equivalent to a suspension map. For (a) an induction could be based on the following

$$
\begin{aligned}
X_{m, 1}^{n} & \sim X_{m, 2}^{n} / X_{m-1,1}^{n} \cup C\left(X_{m-1,1}^{n} \circ S^{n-2}\right), \\
X_{m, j}^{n} / X_{m-1, j-1}^{n} & \cong X_{m, j+1}^{n} / X_{m-1, j}^{n} \cup C\left(X_{m-j, 1}^{n} \circ X_{j, 1}^{n-1}\right) .
\end{aligned}
$$

First $X_{m, m}^{n} / X_{m-1, m-1}^{n} \cong E X_{m, 1}^{n-1}$ and next $X_{m, m-1}^{n} / X_{m-1, m-2}^{n} \sim E K$ by Proposition 2.6 and so on until one reaches $X_{m, 2}^{n} / X_{m-1,1}^{n}$.

3. An important lemma. Recall the attaching map $\varphi: X_{m, 1}^{n} \rightarrow X_{m-1, m-1}^{n+1}=$ $S P^{m-1} S^{n}$ defined in Lemma 2.2. If $f: S P^{m-1} S^{n} \rightarrow S^{n}$ is any map of type $q$ such that $f \varphi$ is nullhomotopic, say by $N_{t}: \varepsilon \sim f \varphi$, then a map $g: S P^{m} S^{n} \rightarrow S^{n}$ of type $q$ is defined by $g \mid S P^{m-1} S^{n}=f$ and $g \mid C X_{m, 1}^{n}=N_{t}$. As $\varphi^{-1}\left(S P^{i} S^{n}\right)=X_{m, m-i}^{n}$ we define maps

$$
\varphi_{i}: X_{m, m-i}^{n} \rightarrow S P^{i} S^{n}, \quad \varphi_{i}^{\prime}: X_{m, m-i}^{n} \cup X_{m-1, m-i-2}^{n} \rightarrow S P^{i+1} S^{n}
$$

by the restrictions $\varphi_{i}=\varphi \mid X_{m, m-i}^{n}$ and $\varphi_{i}^{\prime}=\varphi \mid\left(X_{m, m-i}^{n} \cup X_{m-1, m-i-2}^{n}\right)$.

LeMma 3.1. If $f: S^{n} \rightarrow S^{n}$ is a map of degree $q$ such that $f \varphi_{1}: X_{m, m-1}^{n} \rightarrow S^{n}$ is nullhomotopic, then there exists a map $F: S P^{m-1} S^{n} \rightarrow S^{n}$ of type $q$ such that $F_{\varphi_{m-1}}=F \varphi$ is nullhomotopic. Thus the above construction provides the existence of $a$ map $S P^{m} S^{n} \rightarrow S^{n}$ of type $q$.

Proof. We have a filtration of $X_{m, 1}^{n}$ given by $C_{1} \subset D_{1} \subset C_{2} \subset D_{2} \subset \ldots \subset C_{m-2}$ $\subset D_{m-2} \subset C_{m-1}$ where $C_{i}=X_{m, m-i}^{n}$ and $D_{i}=X_{m, m-i}^{n} \cup X_{m-1, m-i-2}^{n}$, and so we have maps $\varphi_{i}: C_{i} \rightarrow S P^{i} S^{n}$ and $\varphi_{i}^{\prime}: D_{i} \rightarrow S P^{i+1} S^{n}$ determined by $\varphi$. Suppose inductively $f_{i}: S P^{i} S^{n} \rightarrow S^{n}$ is a map of type $q$ with nullhomotopy $N_{i}^{i}: \varepsilon \sim f_{i} \varphi_{i}$. We will then construct a map $f_{i+1}: S P^{i+1} S^{n} \rightarrow S^{n}$ of type $q$ and nullhomotopies $N_{t}^{\prime i}: \varepsilon \sim f_{i+1} \varphi_{i}^{\prime}$ and $N_{t}^{i+1}: \varepsilon \sim f_{i+1} \varphi_{i+1}$. Clearly this will suffice to prove the lemma. The construction of $f_{i+1}, N_{t}^{\prime}$ and $N_{t}$ is based on the geometry of Lemma 2.4:

(i) $C_{i+1} \cong D_{i} \cup C\left(X_{i+1,1}^{n} \circ X_{m-i-1,1}^{n-1}\right)$,

(ii) $D_{i} \cong C_{i} \cup C X_{i+1,1}^{n} \times X_{m-i-2, m-i-2}^{n}$. 
(ii) is an easy consequence of the observation that

$$
C_{i} \cap X_{m-1, m-i-2}^{n}=X_{m-1, m-i-1}^{n} \text {. }
$$

Construction of $f_{i+1}$ : we have the standard inclusion $j: X_{i+1,1}^{n} \subset X_{m, m-i}^{n}$ and so let $M_{t}^{i}=N_{t}^{i} j$ (more precisely $M_{t}^{i}=N_{t}^{i}(j \times \mathrm{id})$ ) be the restriction of the nullhomotopy $N_{t}^{i}$ to $X_{i+1,1}^{n}$. Thus $M_{t}^{i}$ is also a nullhomotopy. But recall $S P^{i+1} S^{n} \cong S P^{i} S^{n}$ $\cup C X_{i+1,1}^{n}$ and so we may define $f_{i+1}$ by $f_{i+1} \mid S P^{i} S^{n}=f_{i}$ and $f_{i+1} \mid C X_{i+1,1}^{n}=M_{t}^{i}$. Because $\varphi_{i} j$ is the attaching map in $S P^{i} S^{n} \cup C X_{i+1,1}^{n}, f_{i+1}$ is well defined.

Construction of the nullhomotopy $N_{u}^{\prime i}: \varepsilon \sim f_{i+1} \varphi_{i}^{\prime}$ : this is simply given by $N_{u}^{\prime i} \mid C_{i}=N_{u}^{i}$ and

$$
N^{\prime i}(([x, t], y), u)=f_{i+1} \varphi_{i}^{\prime}([x, u t], y)
$$

on $C X_{i+1,1}^{n} \times X_{m-i-2, m-i-2}^{n}$. Here $([x, u t], y)$ also denotes a point of $C X_{i+1,1}^{n}$ $\times X_{m-i-2, m-i-2}^{n}$. As $\varphi_{i}^{\prime} \mid\{([x, 0], y)\}$ is the constant map, so is $N_{0}^{\prime i}$. And for $u=1$, $N_{1}^{\prime i}$ is just $f_{i+1} \varphi_{i}^{\prime}$ as desired.

Construction of the nullhomotopy $N_{t}^{i+1}: \varepsilon \sim f_{i+1} \varphi_{i+1}$ : define $N_{t}^{i+1}$ by $N_{t}^{i+1} \mid D_{i}$ $=N_{t}^{\prime i}$ and on $C\left(X_{i+1,1}^{n} \circ X_{m-i-1,1}^{n-1}\right)$ by

(iii) $N^{i+1}\left(\left[\left(x,\left[y, t_{2}\right]\right), s\right], u\right)=M^{i}[x, s u]$,

(iv) $N^{i+1}\left(\left[\left(\left[x, t_{1}\right], y\right), s\right], u\right)=M^{i}\left[x, s t_{1} u\right]$.

The common domain of (iii) and (iv) occurs when $t_{1}=t_{2}=1$ in which case (i) and (ii) reduce to $M^{i}[x, s u]$. When $s=1$ (i) and (ii) are consistent with $N_{t}^{i+1} \mid D^{i}=N_{t}^{\prime i}$. This is clear for $X_{i+1,1}^{n} \times C X_{m-i-1,1}^{n-1}$ since the attaching map of (i) sends $X_{i+1,1}^{n}$ $\times C X_{m-i-1,1}^{n-1}$ to $C_{i}$ and $N_{t}^{\prime i} \mid C_{i}=N_{t}^{i}$. On the other hand $\varphi_{i}^{\prime} \mid C X_{i+1,1}^{n} \times X_{m-i-1,1}^{n-1}$, viewed as a map into $S P^{i} S^{n} \cup C X_{i+1,1}^{n}$, is the identity on $C X_{i+1,1}^{n}$; furthermore $f_{i+1} \mid C X_{i+1,1}^{n}$ is given by $M^{i}$. Thus $N^{\prime i} \mid C X_{i+1,1}^{n} \times X_{m-i-1,1}^{n-1}$ agrees with (iv) above. Finally when $u=0$ we get the constant map and when $u=1$ we get $f_{i+1} \varphi_{i+1}$. This completes the proof of 3.1 .

We conclude this paragraph with a simple observation about the integer $k^{m, n}$ defined in the introduction. Note that $\left[X_{m, m-1}^{n}, S^{n}\right]$ is a distinguished set and the function

$$
\psi_{q}:\left[X_{m, m-1}^{n}, S^{n}\right] \rightarrow\left[X_{m, m-1}^{n}, S^{n}\right]
$$

defined by composition with a $\operatorname{map} f_{q}: S^{n} \rightarrow S^{n}$ of degree $q$, respects distinguished elements. Set $\operatorname{Ker} \psi_{q}=\psi_{q}^{-1}(0)$. Then

Proposition 3.2. Let $n=2 t+1$ and $\varphi, \varphi_{1}, \ldots, \varphi_{m-1}$ be as in 3.1. Then $k^{m, n}$ is the least positive integer $q$ such that $\left[\varphi_{1}\right] \in \operatorname{Ker} \psi_{q}$.

Proof. For any $q>0$ with $\left[\varphi_{1}\right] \in \operatorname{Ker} \psi_{q}$ the preceding lemma implies the existence of a map $f: S P^{m} S^{n} \rightarrow S^{n}$ of type $q>0$, whence $k^{m, n} \mid q$. But by definition of $k^{m, n}$ there exists a map $g: S P^{m} S^{n} \rightarrow S^{n}$ of type $k^{m, n}$. Set $g_{i}=g \mid S P^{i} S^{m}$. Then $g \varphi: X_{m, 1}^{n} \rightarrow S^{n}$ extends over the cone $C X_{m, 1}^{n}$ and so is nullhomotopic. Thus so is $g_{1} \varphi_{1}$. But $g_{1}: S^{n} \rightarrow S^{n}$ has degree $k^{m, n}$ and so $\left[\varphi_{1}\right] \in \operatorname{Ker} \psi_{r}$ for $r=k^{m, n}$. Therefore $q=k^{m, n}$. 
4. Suspension-order. The suspension-order of a space $X$ is the order of the class $\iota_{E X} \in[E X, E X]$ of the identity map of $E X$. In [6] Toda has computed the suspension-order of $E^{r} X_{2,1}^{n}, r \geqq-(n-1)$. Our main concern is the determination of the suspension-order of $E^{r}\left(X_{3,2}^{n} / X_{2,1}^{n}\right), r \geqq-1$. However we begin with an easy result about $E^{r} X_{m, m-1}^{n}, r \geqq-1$.

Proposition 4.1. (i) For $n=2 t$ the suspension-order of $E^{r} X_{m, m-1}^{n}, r \geqq-1$, is infinite.

(ii) For $n=2 t+1$ the suspension-order of $E^{r} X_{m, m-1}^{n}, r \geqq-1$, is a divisor of

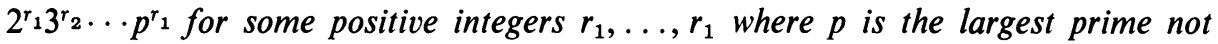
exceeding $m$.

Proof. (i) By Toda [6] we need only prove that $H^{i}\left(X_{m, m-1}^{n} ; Z\right)$ contains an element of infinite order for some $i>0$. We assert that this is the case for $H^{2 n-1}\left(X_{m, m-1}^{n} ; Z\right)$. For $m=2, E X_{2,1}^{n}$ and $S P^{2} S^{n} / S^{n}$ are homeomorphic but

$$
H^{2 n}\left(S P^{2} S^{n} ; Z\right) \cong H^{2 n}\left(S P^{2} S^{n} / S^{n} ; Z\right) \approx Z
$$

is well known.

So assume inductively on $m$ that $H^{2 n-1}\left(X_{m, m-1}^{n} ; Z\right)$ contains an element of infinite order. From Lemma $2.4 X_{m+1, m}^{n}$ is homeomorphic to the adjunction space

$$
\left(X_{m+1, m+1}^{n} \cup X_{m, m-1}^{n}\right) \cup C\left(S^{n-1} \circ X_{m, 1}^{n-1}\right)
$$

(recall $X_{m+1, m+1}^{n}=S P^{m+1} S^{n-1}$ ). As $X_{m+1, m+1}^{n} \cap X_{m, m-1}^{n}=X_{m, m}^{n}=S P^{m} S^{n-1}$ and $H^{i}\left(S P^{m} S^{n-1} ; Z\right)$ is finite for $i=2 n-1,2 n-2$ (Nakaoka [3]), a straightforward application of the cohomology Mayer-Vietoris exact sequence implies that $H^{2 n-1}\left(X_{m+1, m+1}^{n} \cup X_{m, m-1}^{n} ; Z\right)$ contains an element of infinite order. The pair $\left(S P^{m} S^{n-1}, S P^{m-1} S^{n-1}\right) \cong\left(C X_{m, 1}^{n-1}, X_{m, 1}^{n-1}\right)$ is $n$-connected and so the pair (using Lemma 2.4) $\left(X_{m+1, m}^{n}, X_{m+1, m+1}^{n} \cup X_{m, m-1}^{n}\right)$ has trivial integral cohomology in dimensions $0<i<2 n$. And since $n-1$ is odd $H^{2 n}$ is finite. So by the cohomology exact sequence of the pair $\left(X_{m+1, m}^{n}, X_{m+1, m+1}^{n} \cup X_{m, m-1}^{n}\right)$ we obtain that

$$
H^{2 n-1}\left(X_{m+1, m}^{n} ; Z\right)
$$

contains an element of infinite order.

(ii) That $E^{r} X_{m, m-1}^{n}, r \geqq-1$, is simply connected is an easy consequence of (1) $S P^{m} S^{n-1}$ is $(n-2)$-connected, (2) Lemma 2.4, (3) induction on $m$, and (4) the van Kampen Theorem. Hence to prove (ii) it suffices by Theorem 1.5 of [6] to prove that the reduced homology $\tilde{H}^{*}\left(X_{m, m-1}^{n} ; Z\right)$ is finite and has $q$ torsion ( $q$ a prime) exactly when $q \leqq m$. This we do by induction on $m$. For $m=2$ it is well known that $\tilde{H}^{*}\left(X_{2,1}^{n} ; Z\right)$ consists of only 2-torsion. By the cohomology exact sequence of the pair $\left(X_{m+1, m}^{n}, X_{m, m-1}^{n}\right)$ and induction it suffices to prove that

$$
\tilde{H}^{*}\left(X_{m+1, m}^{n}, X_{m, m-1}^{n} ; Z\right)
$$


is finite and has $q$-torsion exactly when $q \leqq m+1$. By Lemma 2.5 we have

$$
\begin{aligned}
X_{m+1, m}^{n} / X_{m, m-1}^{n} & \cong X_{m+1, m+1}^{n} / X_{m, m}^{n} \cup C\left(S^{n-1} \circ X_{m, 1}^{n-1}\right) \\
& \cong E X_{m+1,1}^{n-1} \cup C\left(S^{n-1} \circ X_{m, 1}^{n-1}\right)
\end{aligned}
$$

with the attaching map sending $C S^{n-1} \times X_{m, 1}^{n-1}$ to the basepoint and on $S^{n-1}$ $\times C X_{m, 1}^{n-1}$ given by the composition

$$
\begin{aligned}
S^{n-1} \times C X_{m, 1}^{n-1} & \rightarrow S^{n-1} \times S P^{m} S^{n-1} \\
& \rightarrow S P^{m+1} S^{n-1} \rightarrow S P^{m+1} S^{n-1} / S P^{m} S^{n-1} \cong E X_{m+1,1}^{n-1} .
\end{aligned}
$$

Now rank $\tilde{H}^{*}\left(E X_{m+1,1}^{n-1}, Z\right)=1$, a generator of infinite order occurring in dimension $(m+1)(n-1)$. From the above description of the attaching map this generator is mapped by the coboundary homomorphism onto $(m+1)$-times a generator of infinite order (plus possibly something of finite order) in $H^{i+1}\left(E^{n+1} X_{m, 1}^{n-1} ; Z\right)$, $i=(m+1)(n-1)$. Thus $\tilde{H}^{*}\left(X_{m+1, m}^{n} / X_{m, m-1}^{n} ; Z\right)$ is finite with $q$-torsion, $q \leqq m+1$.

REMARK. The assertion of 4.1 (ii) is also true for $E^{r}\left(X_{m, m-1}^{n} / X_{m-1, m-2}^{n}\right), r \geqq-1$, in place of $E^{r} X_{m, m-1}^{n}$. The proof is essentially the same, but of course the first step is unnecessary.

Lemma 4.2. Let $n=2 t+1$ and $p$ be an odd prime. Then $\left(K U^{1}\right)^{\sim}\left(X_{p, p-1}^{n}\right)$ contains an element of order $p^{t}$.

Proof. Consider the cofibration

$$
X_{p-1, p-2}^{n} \rightarrow X_{p, p-1}^{n} \rightarrow X_{p, p-1}^{n} / X_{p-1, p-2}^{n} .
$$

As $n$ is odd each of these spaces has finite reduced integral cohomology groups. However Nakaoka's results and Proposition 4.1 imply that $\tilde{H}^{*}\left(X_{p-1, p-2}^{n} ; Z\right)$ has $q$-torsion exactly for those primes $q<p$. Hence (via the Atiyah-Hirzebruch spectral sequence) $\left(K U^{*}\right)^{\sim}\left(X_{p-1, p-2}^{n}\right)$ contains no elements of order $p^{r}, r \geqq 1$. Thus we need only prove that $\left(K U^{1}\right)^{\sim}\left(X_{p, p-1}^{n} / X_{p-1, p-2}^{n}\right)$ contains an element of order $p^{t}$.

Similar application of Nakaoka's results shows that the reduced integral cohomology of $E^{n+1} X_{p-1,1}^{n-1} \cong E^{n}\left(S P^{p-1} S^{n-1} / S P^{p-2} S^{n-1}\right)$ has rank 1 and has no $p$-torsion. Again from Lemma 2.5 we have

$$
X_{p, p-1}^{n} / X_{p-1, p-2}^{n} \cong E X_{p, 1}^{n-1} \cup C\left(S^{n-1} \circ X_{p-1,1}^{n-1}\right) .
$$

In addition to $q$-torsion, $q<p, \tilde{H}^{*}\left(E X_{p, 1}^{n-1} ; Z\right)$ has one copy of $Z$ (and that in dimension $(n-1) p)$ and has cyclic $p$-torsion only in dimensions $(n-1)+2 k(p-1)$ $+1, k=1,2, \ldots, t-1$. From the previous description of the attaching map it follows that $\tilde{H}^{*}\left(X_{p, p-1}^{n} / X_{p-1, p-2}^{n} ; Z\right)$ is finite, has $q$-torsion for $q<p$, and has cyclic $p$-torsion only in dimension $(n-1)+2 k(p-1)+1, k=1,2, \ldots, t$.

Now the remaining steps in the proof are a repeat of those given in $\$ 2$ of [7]. Since all the $p$-torsion occurs in odd dimensions, it all survives to $E_{\infty}$. Clearly since there are $t E_{\infty}$-terms (all contributing to $\left(K U^{1}\right)^{\sim}$ ) containing cyclic $p$-torsion 
$\left(K U^{1}\right)^{\sim}\left(X_{p, p-1}^{n} / X_{p-1, p-2}^{n}\right)$ will contain an element of order $p^{t}$ if its $p$-primary part is cyclic. Coefficient $K$-theory and the Universal Coefficient Theorem will suffice for this. $\tilde{H}^{*}\left(X_{p, p-1}^{n} / X_{p-1, p-2}^{n} ; Z_{p}\right)$ consists of $2 t$ copies of $Z_{p}$ and the initial differential $d_{2 p-1}$ (of the Atiyah-Hirzebruch spectral sequence converging to $\left.\left(K U^{*}\right)^{\sim}\left(; Z_{p}\right)\right)$ kills all but two copies of $Z_{p}$, one in an odd dimension and the other in an even dimension. The Universal Coefficient Theorem then implies that $\left(K U^{1}\right)^{\sim}\left(X_{p, p-1}^{n} / X_{p-1, p-2}^{n}\right)$ is cyclic. This completes the proof of 3.2.

For the suspension-order determination of $X_{3,2}^{n} / X_{2,1}^{n}$ and $X_{3,2}^{n}$ we require more precise information on $\tilde{H}^{*}\left(X_{3,2}^{n} / X_{2,1}^{n} ; Z\right)$ and $\tilde{H}^{*}\left(X_{3,2}^{n} ; Z\right)$.

LEMMA 4.3. For $n=2 t+1$,

$$
\begin{aligned}
\tilde{H}^{i}\left(X_{3,2}^{n} / X_{2,1}^{n} ; Z\right) & \cong Z_{3} \text { if } i=(n-1)+4 k+1, k=1,2, \ldots, t, \\
& \cong 0 \quad \text { otherwise. }
\end{aligned}
$$

Proof. We start with the familiar

$$
X_{3,2}^{n} / X_{2,1}^{n} \cong S P^{3} S^{n-1} / S P^{2} S^{n-1} \cup C\left(S^{n-1} \circ X_{2,1}^{n-1}\right) .
$$

Nakaoka [2] has shown that $H^{*}\left(S P^{3} S^{n-1}, S P^{2} S^{n-1} ; Z\right) \cong H^{*}\left(E^{n} X_{2,1}^{n-1} ; Z\right) \oplus K$ where $K=K_{1} \oplus \cdots \oplus K_{t}, K_{i} \cong Z_{3}$ and dimension $K_{i}=(n-1)+4 i+1$.

Thus in the commutative diagram

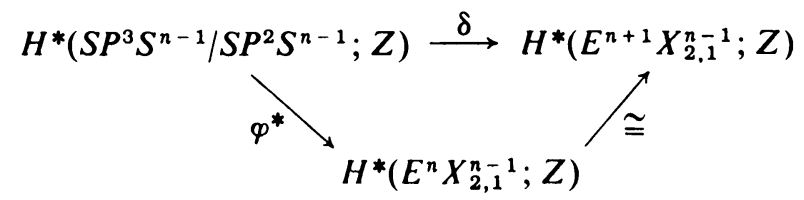

we need only prove that $\varphi^{*}$ is an isomorphism when restricted to the direct summand $H^{*}\left(E^{n} X_{2,1}^{n-1} ; Z\right)$.

Now $\varphi$ factors as

$$
\begin{aligned}
S^{n-1} \circ X_{2,1}^{n-1} \longrightarrow \frac{S^{n-1} \circ X_{2,1}^{n-1}}{A} & \cong \frac{S^{n-1} \times C X_{2,1}^{n-1}}{\text { point } \times C X_{2,1}^{n-1} \cup S^{n-1} \times X_{2,1}^{n-1}} \\
& \cong \frac{S^{n-1} \times S P^{2} S^{n-1}}{S^{n-1} \vee S P^{2} S^{n-1}} \stackrel{\bar{\varphi}}{\longrightarrow} \frac{S P^{3} S^{n-1}}{S P^{2} S^{n-1}}
\end{aligned}
$$

where $\bar{\varphi}$ is induced by the composite

$$
S^{n-1} \times S P^{2} S^{n-1} \stackrel{p_{1}}{\longrightarrow} S P^{3} S^{n-1} \stackrel{p_{2}}{\longrightarrow} S P^{3} S^{n-1} / S P^{2} S^{n-1}
$$

But $p_{1}^{*} u=u_{1}+u_{2}$ where $u, u_{1}, u_{2}$ are $(n-1)$-dimensional cohomology generators (of infinite order). By Nakaoka [2] the direct summand $\tilde{H}^{*}\left(E^{n} X_{2,1}^{n-1} ; Z\right)$ of $H^{*}\left(S P^{3} S^{n-1} / S P^{2} S^{n-1} ; Z\right)$ is represented by the classes $u \cdot\left(\delta_{2} S q^{2 i} u\right)$ and so $\varphi^{*}\left(u \cdot \delta_{2} S q^{2 i} u\right)=u_{1} \otimes \delta_{2} S q^{2 i} u$. But $H^{*}\left(E^{n} X_{2,1}^{n-1} ; Z\right)$ lies in the image of $p_{2}^{*}$ and so the result follows. 
LeMma 4.4. Let $n=2 t+1$. The exact cohomology sequence of the pair $\left(X_{3,2}^{n}, X_{2,1}^{n}\right)$ breaks up into short exact sequences, and so

$$
\tilde{H}^{*}\left(X_{3,2}^{n} ; Z\right) \cong \tilde{H}^{*}\left(X_{2,1}^{n} ; Z\right) \oplus \tilde{H}^{*}\left(X_{3,2}^{n} / X_{2,1}^{n} ; Z\right)
$$

Proof. We have a commutative diagram

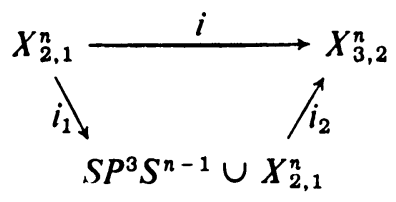

Since $X_{3,2}^{n} /\left(S P^{3} S^{n-1} \cup X_{2,1}^{n}\right) \cong E^{n+1} X_{2,1}^{n}$ is $(2 n+1)$-connected, $i_{2}^{*}$ is an isomorphism in dimensions $\leqq 2 n-1$. But a straightforward application of the Mayer-Vietoris cohomology exact sequence (note $S P^{3} S^{n-1} \cap X_{2,1}^{n}=S P^{2} S^{n-1}$ ) shows that $i_{1}^{*}$ is epimorphic. And so $i^{*}$ is epimorphic and the result follows.

Lemma 4.5. Let $n=2 t+1$. Then $\left(K O^{n}\right)^{\sim}\left(X_{3,2}^{n}\right)$ contains an element of order $2^{\varphi(2 t)}$.

Proof. Consider the exact sequence

$$
\left(K O^{n}\right) \sim\left(X_{3,2}^{n}\right) \rightarrow\left(K O^{n}\right) \sim\left(X_{2,1}^{n}\right) \rightarrow\left(K O^{n+1}\right) \sim\left(X_{3,2}^{n}, X_{2,1}^{n}\right) .
$$

From Lemma 4.3 and the Atiyah-Hirzebruch spectral sequence

$$
\left(K O^{n+1}\right) \sim\left(X_{3,2}^{n}, X_{2,1}^{n}\right)
$$

has no 2-torsion. But $\left(K O^{n}\right)^{\sim}\left(X_{2,1}^{n}\right) \cong Z_{2}^{\omega(2 t)}$ since $X_{2,1}^{n}$ and $E^{n} P^{n-1}$ are homeomorphic (actually all one needs for this particular situation is that $H^{*}\left(X_{2,1}^{n} ; Z\right) \cong H^{*}\left(E^{n} P^{n-1} ; Z\right)$ as modules over the Steenrod algebra) and so the result follows by exactness.

TheOREM 4.6. Let $n=2 t+1$. The suspension-order of $E^{r}\left(X_{3,2}^{n} / X_{2,1}^{n}\right), r \geqq-1$, is $3^{t}$.

Proof. It follows from Lemma 4.2 (for $p=3$ ) and Toda [6] that the suspensionorder of $E^{r}\left(X_{3,2}^{n} / X_{2,1}^{n}\right), r \geqq-1$, is a multiple of $3^{t}$. So we need only show that it is also a divisor of $3^{t}$. By Lemma 1.3 of [6] and our Lemma 4.3 there is a sequence of subcomplexes $*=L_{1} \subset L_{2} \subset \ldots \subset L_{r-1} \subset L_{r} \subset \ldots \subset L=E^{r}\left(X_{3,2}^{n} / X_{2,1}^{n}\right)$ with $L_{i}$ $=L_{i-1} \cup e^{i} \cup e^{i+1}$ or $L_{i}=L_{i-1}$ according as $i-r=n-1+4 k(k=1,2, \ldots, t)$ or not. Set $K_{j}=L_{i}$ for $i-r=n-1+4 j$ so we have $* \subset K_{1} \subset K_{2} \subset \ldots \subset K_{t}$ and $K_{j}=K_{j-1}$ $\cup e^{i} \cup e^{i+1}$.

By Lemma 1.4 of [6] and the second paragraph of the proof of Theorem 4.4 of [6] the class $3 \iota_{E Z}, Z=E^{r}\left(X_{3,2}^{n} / X_{2,1}^{n}\right)$, has a representative $f$ satisfying $f\left(E K_{j}\right) \subset E K_{j-1}$. So the class $3^{t} t_{E Z}$ is represented by the map $\hat{f}=f \circ f \circ \cdots \circ f(t$ times $)$ which satisfies $\hat{f}\left(E K_{t}\right)=*$ i.e. $\hat{f}$ is nullhomotopic. Whence the suspension-order of $Z$ is a divisor of $3^{t}$.

For completeness we include 
THEOREM 4.7. Let $n=2 t+1$. The suspension-order of $E^{r} X_{3,2}^{n}, r \geqq-1$, is $2^{\varphi(2 t)} \cdot 3^{t}$.

Proof. First by Lemmas 4.2 and 4.5 and Toda's Theorem 1.1 [6] the suspensionorder of $E^{r} X_{3,2}^{n}$ is a multiple of $2^{\varphi(2 t)} \cdot 3^{t}$. To prove it is also a divisor of this number consider the fibration

$$
X_{2,1}^{n} \rightarrow X_{3,2}^{n} \rightarrow X_{3,2}^{n} / X_{2,1}^{n} .
$$

By the corollary to Theorem 2.6 of [6] the suspension-order of $E^{r} X_{2,1}^{n}, r \geqq-1$, is a divisor of $2^{\varphi(2 t)}$. And from Lemma 4.6 above the suspension-order of $E^{r}\left(X_{3,2}^{n} / X_{2,1}^{n}\right)$ is a divisor of $3^{t}$. So by Theorem 1.2 of [6] we have that the suspension-order of $E^{r} X_{3,2}^{n}$ is a divisor of $2^{\varphi(2 t)} \cdot 3^{t}$.

5. The mod 3 Hopf invariant. The generalized Hopf invariant is a homomorphism

for which the sequence

$$
\left[E K, S^{n}\right] \stackrel{H}{\longrightarrow}\left[E K, S^{2 n-1}\right]
$$

$$
\left[K, S^{n-1}\right] \stackrel{E}{\longrightarrow}\left[E K, S^{n}\right] \stackrel{H}{\longrightarrow}\left[E K, S^{2 n-1}\right]
$$

is exact when dimension $E K \leqq 3(n-1)$. $H$ also satisfies the property $\psi_{q^{2}} H=H \psi_{q}$. In $\S 4$ of [1] $H$ is used to prove $\psi_{4 r}[h]=4 r[h]$ for any $[h] \in\left[X_{2,1}^{n}, S^{n}\right], n=2 t+1 \geqq 3$.

Our situation is analogous but with one important exception: the dimension restriction is not applicable. So we consider the $\bmod p$ Hopf invariant $H_{p}, p$ an odd prime (we assume the reader is familiar with the relevant sections of [5]). $H_{p}$ is defined by the commutative diagram

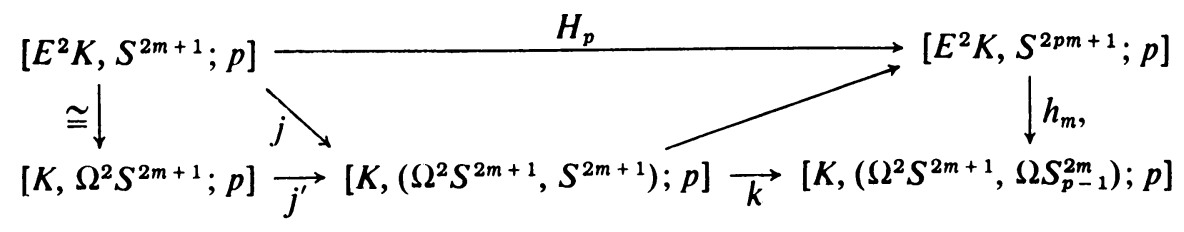

where $[; p]$ denotes the $p$-primary component. It is known that $h_{m}$, is an isomorphism and that $\operatorname{Ker} j$ is the $p$-primary component of image $E^{2}$ where $E^{2}:\left[K, S^{2 m-1}\right] \rightarrow\left[E^{2} K, S^{2 m+1}\right]$. So whenever $k$ is a monomorphism, we will have $\operatorname{Ker} H_{p}=\operatorname{Ker} j=p$-primary component of image $E^{2} . k$ occurs in the homotopy exact sequence of the triple $\left(\Omega^{2} S^{2 m+1}, \Omega S_{p-1}^{2 m}, S^{2 m-1}\right)$ as

$$
\begin{aligned}
{\left[K,\left(\Omega S_{p-1}^{2 m}, S^{2 m-1}\right) ; p\right] } & \longrightarrow\left[K,\left(\Omega^{2} S^{2 m+1}, S^{2 m-1}\right) ; p\right] \\
& \stackrel{k}{\longrightarrow}\left[K,\left(\Omega^{2} S^{2 m+1}, \Omega S_{p-1}^{2 m}\right) ; p\right] .
\end{aligned}
$$

The group on the left is isomorphic to $\left[E K, S^{2 p m-1} ; p\right]$ so whenever the latter is trivial, $k$ is a monomorphism. We use this observation in 5.2 , but first we have

Lemma 5.1. Suppose $H^{i}\left(E^{2} K ; Z\right)=0$ for all $i>p n-(p-1), n=2 m+1$, and $q H^{p n-(p-1)}\left(E^{2} K ; Z\right)=0$ for some integer $q$. 
Then $\operatorname{Ker} H_{p}$ contains that the subgroups $q\left[E^{2} K, S^{2 m+1} ; p\right]$ and $\psi_{q}\left[E^{2} K, S^{2 m+1} ; p\right]$. So

Proof. By the Hopf Theorem we have $\left[E^{2} K, S^{p n-(n-1)}\right] \cong H^{p n-(n-1)}\left(E^{2} K ; Z\right)$.

$$
\begin{aligned}
H_{p} q\left[E^{2} K, S^{2 m+1} ; p\right] & =q H_{p}\left[E^{2} K, S^{2 m+1} ; p\right] \subset q\left[E^{2} K, S^{2 p m+1}\right] \\
& =q\left[E^{2} K, S^{p n-(n-1)}\right]=0 .
\end{aligned}
$$

And since $H_{p} \psi_{q}=\psi_{q^{p}} H_{p}$ we have

$$
H_{p} \psi_{q}\left[E^{2} K, S^{2 m+1} ; p\right]=\psi_{q^{p}} H_{p}\left[E^{2} K, S^{2 m+1} ; p\right] \subset \psi_{q^{p}}\left[E^{2} K, S^{2 p m+1}\right]=0
$$

since $\psi_{q^{p}}$ acts on $\left[E^{2} K, S^{2 p m+1}\right] \cong H^{2 p m+1}\left(E^{2} K ; Z\right)$ as multiplication by $q^{p}$.

Lemma 5.2. Let $r=k q^{2}$ and assume $\left[E K, S^{2 p m-1} ; p\right]=0$ in addition to the hypotheses of 5.1. Then $\psi_{r}$ acts on $\left[E^{2} K, S^{2 m+1} ; p\right]$ as multiplication by $r$.

Proof. For $\alpha \in\left[E^{2} K, S^{2 m+1} ; p\right]$ we have $\psi_{r} \alpha=\psi_{k q}\left(\psi_{q} \alpha\right)=k q\left(\psi_{q} \alpha\right)=k \psi_{q}(q \alpha)=$ $k q(q \alpha)=r \alpha$ using 5.1 and the fact that $\operatorname{Ker} H_{p}=\operatorname{Image} E^{2}$ when $\left[E K, S^{2 p m-1} ; p\right]=0$.

Proof of Theorem 1.2(ii). For $n=1$ the result is given by the group structure on $S^{1} \cong U(1)$. For $n=2 t+1 \geqq 3$ we consider the cofibration

$$
X_{2,1}^{n} \longrightarrow X_{3,2}^{n} \stackrel{p}{\longrightarrow} X_{3,2}^{n} / X_{2,1}^{n}
$$

and the associated exact sequence

$$
\left[X_{3,2}^{n} / X_{2,1}^{n}, S^{n}\right] \stackrel{p^{*}}{\longrightarrow}\left[X_{3,2}^{n}, S^{n}\right] \stackrel{i^{*}}{\longrightarrow}\left[X_{2,1}^{n}, S^{n}\right] .
$$

The main results of [1] and [6] imply that $i^{*} \psi_{r} \alpha=0$ for $r=2^{\varphi(2 t)}$ and so by exactness we obtain an element $\beta \in\left[X_{3,2}^{n} / X_{2,1}^{n}, S^{n}\right]$ with $p^{*} \beta=\psi_{r} \alpha$. So it is enough to show that $\psi_{s} \beta=0$ for $s=3^{t}$.

For $n=3$ we have from Lemma 2.5 that $X_{3,2}^{3} / X_{2,1}^{n}$ is homeomorphic to the complex $S^{6} \cup_{f} e^{7}$, degree $f=3$-note $C S^{2} \times C X_{2,1}^{2}$ is just a 7-cell since $X_{2,1}^{2}$ is a 3-cell. Hence the only obstructions to nullhomotoping a given map $S^{6} \cup_{f} e^{7} \stackrel{g}{\rightarrow} S^{3}$ lie in $H^{6}\left(S^{6} \cup_{f} e^{7} ; \pi_{6}\left(S^{3}\right)\right) \cong Z_{3}$ and $H^{7}\left(S^{6} \cup_{f} e^{7} ; \pi_{7}\left(S^{3}\right)\right)=0$. But $\psi_{3}: \pi_{6}\left(S^{3}\right) \rightarrow \pi_{6}\left(S^{3}\right)$ is multiplication by 3 (since $E: \pi_{6}\left(S^{3}\right) \rightarrow \pi_{7}\left(S^{4}\right)$ is a monomorphism, or alternatively since $\pi_{6}\left(S^{3}\right)=\pi_{6}\left(S^{3} ; 3\right)$ and we can invoke (13.13) of [5]). Thus $\psi_{3} \beta=0$ as required.

For $n=2 t+1 \geqq 5$ we can apply 5.2 whenever the condition

$$
\left[E^{-1}\left(X_{3,2}^{n} / X_{2,1}^{n}\right), S^{6 t-1} ; 3\right]=0
$$

obtains-in which case we again obtain the desired result $\psi_{s} \beta=s \beta=0$ for $s=3^{t}$ (using both Theorem 1.4(ii) and Lemma 5.2). Now $H^{i}\left(E^{-1}\left(X_{3,2}^{n} / X_{2,1}^{n}\right) ; Z\right)=0$ for $i=3 n-4$ or $i>3 n-3$, and is $\cong Z_{3}$, for $i=3 n-3$. As dimension $E^{-1}\left(X_{3,2}^{n} / X_{2,1}^{n}\right)=6 t$ the Steenrnd Classification Theorem $[4$, p. 460$]$ immediately implies that

$$
\left[E^{-1}\left(X_{3,2}^{n} / X_{2,1}^{n}\right), S^{6 t-1}\right]=0 .
$$


Remarks. 1. It is possible to show that the suspension-order of $E^{r} X_{3,1}^{n}, r \geqq-1$ and $n=2 t+1$, is also $2^{\varphi(2 t)} 3^{t}$. Only minor modification of the hypotheses of Toda's Lemma 1.4 is needed for this. Indeed this was our first approach. However Lemma 3.1 showed that the suspension-order of $E^{r}\left(X_{3,2}^{n} / X_{2,1}^{n}\right)$ would be more useful.

2. We conjecture that the lower bound for $k^{m, n}$ given in [7] is not best possible for any value $m>3$, in particular for $m=4$. It is really just a first-order result. Although we feel that $K$-theory will provide the best possible lower bound for all $m$, higher-order considerations (in the use of coefficients $K$-theory) must enter for $m>3$. Finally we conjecture that for each prime $p$, the largest power $p^{v}$ of $p$ dividing $k^{m, n}$ is an unbounded, nondecreasing function of $m$ (for fixed $n=2 t+1 \geqq 3$ ). The lower bound of [7] does not reflect this kind of behavior.

\section{REFERENCES}

1. I. M. James, Emery Thomas, H. Toda and G. W. Whitehead, On the symmetric square of a sphere, J. Math. Mech. 12 (1963), 771-776. MR 27 \#4231.

2. M. Nakaoka, Cohomology theory of a complex with a transformation of prime period and and its applications, J. Inst. Polytech. Osaka City Univ. Ser. A 7 (1956), 51-102. MR 19, 972.

3. - Cohomology mod $p$ of symmetric products of spheres. II, J. Inst. Polytech. Osaka City Univ. Ser. A 10 (1959), 67-89. MR 22 \#12519c.

4. E. H. Spanier, Algebraic topology, McGraw-Hill, New York, 1966. MR 35 \#1007.

5. H. Toda, Composition methods in homotopy groups of spheres, Ann. of Math. Studies, no. 49, Princeton Univ. Press, Princeton, N. J., 1962. MR 26 \#777.

6. - Order of the identity class of a suspension space, Ann. of Math. (2) 78 (1963), 300-325. MR 27 \#6271.

7. J. J. Ucci, On symmetric maps of spheres, Invent. Math. 5 (1968), 8-18. MR 37 \#917.

Forschungsinstitut für Mathematik, Eidgenössosche TeChnische Hochschule, ZÜRICH, SWITZERLAND

INDIANA UNIVERSITY,

BLOOMINGTON, INDIANA 47401 